\title{
Google Scholar, Microsoft Academic, Scopus, Dimensions, Web of Science, and OpenCitations' COCl: a multidisciplinary comparison of coverage via citations
}

\author{
Alberto Martín-Martín ${ }^{1}$ (i) $\cdot$ Mike Thelwall $^{2}$ (D) - Enrique Orduna-Malea ${ }^{3}$ (D) \\ Emilio Delgado López-Cózar ${ }^{1}$ (D)
}

Received: 30 April 2020 / Published online: 21 September 2020

(c) Akadémiai Kiadó, Budapest, Hungary 2020, corrected publication 2020

\begin{abstract}
New sources of citation data have recently become available, such as Microsoft Academic, Dimensions, and the OpenCitations Index of CrossRef open DOI-to-DOI citations (COCI). Although these have been compared to the Web of Science Core Collection (WoS), Scopus, or Google Scholar, there is no systematic evidence of their differences across subject categories. In response, this paper investigates 3,073,351 citations found by these six data sources to 2,515 English-language highly-cited documents published in 2006 from 252 subject categories, expanding and updating the largest previous study. Google Scholar found $88 \%$ of all citations, many of which were not found by the other sources, and nearly all citations found by the remaining sources (89-94\%). A similar pattern held within most subject categories. Microsoft Academic is the second largest overall (60\% of all citations), including $82 \%$ of Scopus citations and $86 \%$ of WoS citations. In most categories, Microsoft Academic found more citations than Scopus and WoS (182 and 223 subject categories, respectively), but had coverage gaps in some areas, such as Physics and some Humanities categories. After Scopus, Dimensions is fourth largest (54\% of all citations), including $84 \%$ of Scopus citations and $88 \%$ of WoS citations. It found more citations than Scopus in 36 categories, more than WoS in 185, and displays some coverage gaps, especially in the Humanities. Following WoS, COCI is the smallest, with $28 \%$ of all citations. Google Scholar is still the most comprehensive source. In many subject categories Microsoft Academic and Dimensions are good alternatives to Scopus and WoS in terms of coverage.
\end{abstract}

Keywords Google Scholar · Microsoft Academic · Scopus · Dimensions · Web of Science · OpenCitations · COCI · CrossRef · Coverage - Citations · Bibliometrics · Citation analysis · Bibliographic databases $\cdot$ Literature reviews

Alberto Martín-Martín

albertomartin@ugr.es

1 Facultad de Comunicación y Documentación, Universidad de Granada, Granada, Spain

2 Statistical Cybermetrics Research Group, School of Mathematics and Computer Science, University of Wolverhampton, Wolverhampton, UK

3 Universitat Politècnica de València, Valencia, Spain 


\section{Introduction}

\section{Timeline}

The first scientific citation indexes were developed by the Institute for Scientific Information (ISI). The Science Citation Index (SCI) was introduced in 1964, and was later joined by the Social Sciences Citation Index (1973) and the Arts \& Humanities Citation Index (1978). In 1997, these citation indexes were moved online under the name "Web of Science". Recently, these citation indexes, along with some new ones such as the Conference Proceedings Citation Index, the Book Citation Index, and the Emerging Sources Citation Index, were rebranded as the "Web of Science Core Collection" (from now on, WoS). The availability of this data was essential to the development of quantitative studies of science as a field of study (Birkle et al. 2020).

In November 2004, two new academic bibliographic data sources that contained citation data were launched. Like WoS, Elsevier's Scopus is a subscription-based database with a selective approach to document indexing (documents from a pre-selected list of publications). A few weeks after Scopus, the search engine Google Scholar was launched. Unlike WoS and Scopus, Google Scholar follows an inclusive and automated approach, indexing any seemingly academic document that its crawlers can find and access on the web, including those behind paywalls through agreements with their publishers (Van Noorden 2014). Additionally, Google Scholar is free to access, allowing users to access a comprehensive and multidisciplinary citation index without charge.

In 2006, Microsoft launched Microsoft Academic Search but retired it in $2012^{1}$ (Orduña-Malea et al. 2014). In 2016, Microsoft launched a new platform called Microsoft Academic, based on Bing's web crawling infrastructure. Like Google Scholar, Microsoft Academic is a free academic search engine, but unlike Google Scholar, Microsoft Academic facilitates bulk access to its data via an Applications Programming Interface (API) (Wang et al. 2020).

In 2018, Digital Science launched the Dimensions database (Hook et al. 2018). Dimensions uses a freemium model in which the basic search and browsing functionalities are free, but advanced functionalities, such as API access, require payment. This fee can be waived for non-commercial research projects.

Also in 2018, the organization OpenCitations, dedicated to developing an open research infrastructure, released the first version of its COCI dataset (OpenCitations Index of CrossRef open DOI-to-DOI citations). The citation data in COCI comes from the lists of references openly available in CrossRef (Heibi et al. 2019). Until 2017, most publishers did not make these references public, but the Initiative for Open Citations (I4OC), launched in April 2017, has since convinced many publishers to do so. The rationale is that citation data should be considered a part of the commons and should not be only on the hands of commercial actors (Shotton 2013, 2018). At the time of writing, 59\% of the 47.6 million articles with references deposited with CrossRef have their references open. ${ }^{2}$ However, some large publishers, such as Elsevier, the American Chemical Society, and IEEE have not yet agreed to opening their lists of references. Thus, COCI's only partially reflects the

\footnotetext{
${ }^{1}$ https://web.archive.org/web/20170105184616/https:/academic.microsoft.com/FAQ

${ }^{2}$ https://i4oc.org/.
} 
citation relationships of documents recorded in CrossRef, which now covers over 106 million records (Hendricks et al. 2020).

The new bibliographic data sources are changing the landscape of literature search and bibliometric analyses. The openly available data in Microsoft Academic Graph (MAG) has been integrated into other platforms, significantly increasing their coverage (Semantic Scholar, Lens.org). There are still some reuse limitations, such as that the current license of MAG (ODC-BY) requires attribution, which apparently precludes it from being able to be integrated into $\mathrm{COCI}$ (which uses a $\mathrm{CC} 0$ public domain license). This openness is nevertheless an advance on the previous situation, in which most citation data was either not freely accessible (WoS, Scopus), or free but with significant access restrictions (Google Scholar). At this point, citation data is starting to become ubiquitous, and even owners of closed bibliographic sources, such as Scopus, are beginning to offer researchers options to access their data for free. ${ }^{3}$

Other citation indexes have been developed within various academic platforms, but these are not analysed in this study, for various reasons:

- CiteSeerX, ${ }^{4}$ from Penn State University, indexes documents in the public web and not those that are only found behind paywalls (Wu et al. 2019).

- ResearchGate ${ }^{5}$ generates its own citation index based on the full text documents that its crawler finds on the Web and those that its users upload to the platform. However, the platform does not offer a way to extract data in bulk, and it is difficult to use web scraping to obtain data because the complete list of citations to an article cannot be easily displayed.

- Lens.org ${ }^{6}$ integrates coverage from Microsoft Academic, CrossRef, PubMed, and a number of Patent datasets. It is not included in this analysis because two of its main sources (Microsoft Academic and CrossRef) are already included.

- Semantic Scholar ${ }^{7}$ originally focused on Computer Science and Engineering. Later it expanded to include Biomedicine, and recently it has integrated multidisciplinary coverage from Microsoft Academic (which is also the reason why we decided not to analyse it).

- There are also several regional or subject-specific citation indexes, which only index documents published by journals and/or researchers who work in a specific country or region, or in specific topics. Given their specific scope these are not easily comparable to sources with a worldwide and/or multidisciplinary coverage.

\section{Previous analyses of coverage}

Document coverage varies across data sources (Ortega 2014), and studies that analyse differences in coverage can inform prospective users about the comprehensiveness of each database in different subject areas. For citation indexes, greater coverage should equate to higher citation counts for documents, if citations can be extracted from all documents.

\footnotetext{
3 https://www.elsevier.com/icsr/icsrlab.

${ }^{4}$ https://citeseerx.ist.psu.edu/index.

5 https://www.researchgate.net/.

6 https://www.lens.org/.

7 https://www.semanticscholar.org/.
} 
Coverage is not the only relevant aspect that should be considered when deciding which data source should be used for a specific information need (e.g., literature search, data for bibliometric analyses). Other aspects such as functionalities to search, analyse, and export data, as well as transparency and cost, are also relevant, but not analysed here. Some of these aspects are analysed by Gusenbauer and Haddaway (2020).

\section{The veterans: WoS, Scopus, and Google Scholar}

As the longest-running platforms, many studies have analysed the differences in coverage and citation data between WoS, Scopus, and Google Scholar. WoS covers over 75 million records in its Core Collection (which includes its main citation indexes), and up to 155 million records when other regional and subject-specific citation indexes are included (Birkle et al. 2020). Scopus claims to cover over 76 million records (Baas et al. 2020). Google Scholar does not disclose official figures about its coverage (Van Noorden 2014), but the most recent independent studies have estimated that it covers well over 300 million records (Delgado López-Cózar et al. 2019; Gusenbauer 2018). At this point most studies agree that Google Scholar has a more comprehensive coverage than Scopus and WoS, and includes the great majority of the documents that they cover. However, the relatively low quality of the metadata available in Google Scholar and the difficulty to extract it make it challenging to use Google Scholar data in bibliometric analyses (Delgado López-Cózar et al. 2019; Halevi et al. 2017; Harzing 2016a, b; Harzing and Alakangas 2016; Martín-Martín et al. 2018; Moed et al. 2016).

\section{Microsoft academic}

Microsoft Academic has been recently reported to cover over 225 million publications (Wang et al. 2020). Harzing carried out an analysis of her own publication record and the publication records of 145 academics in five broad disciplinary areas (Harzing 2016a, b; Harzing and Alakangas 2017a, b). Microsoft Academic found more of her own publications than Scopus or WoS. For the sample of publications by 145 academics, Microsoft Academic provided higher citation counts than both Scopus or WoS in Engineering, Social Sciences, and the Humanities, and similar figures in Life Sciences and Sciences. Google Scholar reported the highest citation counts in all disciplines.

Hug and Brändle (2017) also analysed the coverage of Microsoft Academic and compared it to Scopus and WoS. Based on publications included in the repository of the University of Zurich as a case study, Microsoft Academic had wider coverage of non-article documents than Scopus and WoS, while Scopus had a slightly lower coverage of journal articles than Microsoft Academic. Microsoft Academic showed similar biases to Scopus and WoS against non-English publications and publications in the Humanities. Haunschild et al. (2018) analysed a subset of the same sample used in the previous study $(25,539$ papers also covered by $\mathrm{WoS}$ ) and found that $11 \%$ had no associated cited references in Microsoft Academic, while in WoS the same papers had associated cited references. However, for publications with less than 50 associated references in WoS $(24,788)$ the concordance correlation coefficient applied to the number of references found by each source was 0.68 , indicating a strong tendency for them both to report the same number.

Thelwall (2017) analysed the citation counts of 172,752 articles in 29 large journals from various disciplines, and compared them to Scopus citation counts and Mendeley reader counts. For articles published between 2007 and 2017, Microsoft Academic found 
slightly more citations than Scopus overall, and significantly more than Scopus for documents published in 2017. In subsequent studies, Thelwall (2018a) found that Microsoft Academic did find earlier citations to recently published articles when compared to Scopus. Kousha and Thelwall (2018) studied the coverage and citation counts of books in Microsoft Academic and Google Books by analysing a sample of book records extracted from the Book Citation Index (BKCI) in WoS. They found 60\% of the books in their sample overall, but this percentage was lower in some categories of the Humanities and Social Sciences. Citation counts in Microsoft Academic were higher than in BKCI in 9 out of 17 fields during 2013-2016. Kousha et al. (2018) analysed whether Microsoft Academic was able to find early citations of in-press articles using a sample of 65,000 in-press articles from 2016 to 2017, and found that Microsoft Academic was able to find 2-5 times as many citations as Scopus. This was mostly because Microsoft Academic (like Google Scholar) merges preprints (and the citations these receive) with their subsequent in-press versions, and because Microsoft Academic covers repositories such as arXiv.

Visser et al. (2020) carried out a large-scale comparison of WoS, Scopus, Dimensions, Microsoft Academic, and CrossRef by matching the entire collection of documents in each source. They found that Microsoft Academic was the source with the largest coverage overall, and the one with the higher overlap with Scopus documents (81\% of Scopus documents were found in Microsoft Academic). Some of the documents in Microsoft Academic were not of a scientific nature. Microsoft Academic was not able to detect $12.7 \%$ of the citations found by Scopus after adjusting for coverage differences.

\section{Dimensions}

Dimensions covers over 105 million publications, as well as other kinds of records such as grants data, clinical trials, patents, and policy documents (Herzog et al. 2020).

Orduña-Malea \& Delgado-López-Cózar (2018) analysed several small samples of journals, documents and authors in the field of Library \& Information Science using Dimensions, and compared the data to Scopus and Google Scholar. Dimensions provided slightly lower citation counts than Scopus. Thelwall (2018c) analysed a random sample of 10,000 Scopus articles from 2012, finding that Dimensions covered the great majority of articles with a DOI (97\%) and high correlations between citation counts in the two sources (median of 0.96 across narrow subject categories).

Harzing (2019) analysed coverage of Dimensions and CrossRef, and compared it to the coverage in WoS, Scopus, Google Scholar, and Microsoft Academic using her own publication and citation record, as well as that of six top journals in Business \& Economics. CrossRef and Dimensions had similar or better coverage of publications, and similar citation counts to those in WoS and Scopus, but still substantively lower than Google Scholar and Microsoft Academic.

Visser et al. (2020) found that Dimensions had a substantially higher coverage than Scopus and WoS, which heavily relied on data from CrossRef. After computing the overlap in coverage between Dimensions and Scopus, they found that overall, Dimensions covered $78 \%$ of the documents available in Scopus (35.1 million out of 44.9 million documents in Scopus). They also analysed the accuracy and completeness of citation links, finding that, after adjusting for coverage differences, there were 489.7 million citations found by both sources (percentage of full overlap: 83\%), 73.2 million only found by Scopus, and 25.8 million only found by Dimensions. 


\section{$\mathrm{COCl}$}

COCI has detected over 624 million citation relationships involving over 53 million documents (Peroni and Shotton 2020). The citations recorded in this source are only a fraction of the citations that have actually occurred among the documents covered by CrossRef, because some publishers that deposit lists of references or CrossRef have not agreed to make them available, and other publishers and preprint servers do not deposit any references in CrossRef or do it only for some document types (Shotton 2018; van Eck et al. 2018). Huang et al. (2020) used citation data from COCI and bibliographic data from WoS, Scopus and Microsoft Academic to test the robustness of university rankings created with these different sources, and concluded that despite its lack of comprehensiveness COCI is already a viable data source for cross comparisons at the system level.

\section{Objective}

The citation index coverage studies published so far have analysed a heterogeneous variety of samples of documents, disciplines, and data sources. In response, this paper reports a systematic comparison of coverage of six data sources (Google Scholar, Microsoft Academic, Scopus, Dimensions, $\mathrm{WoS}$, and $\mathrm{COCI}^{8}$ ) across 252 subject categories using a relatively large sample of citations. This allows comparisons across a large number of disciplines for the most widely used bibliographic data sources. This study expands and updates a previous analysis of Google Scholar, Scopus and WoS (Martín-Martín et al. 2018). The main research question that drives this is investigation is:

$R Q$ How much overlap is there between Google Scholar, Microsoft Academic, Scopus, Dimensions, WoS, and COCI in the citations that they find to academic documents and does this vary by subject?

\section{Methods}

\section{Direct coverage comparison versus comparison of citations}

The most direct method to compare document coverage across different data sources would be to obtain a complete list of all documents covered by each source, match the documents across databases, and report the size of the overlaps (Visser et al. 2020). This is not possible here because of access restrictions. For example, Scopus and WoS charge for this kind of access and Google Scholar does not share its database.

Because of these restrictions, studies analysing coverage differences across bibliographic data sources often use an alternative method: they select a seed sample of documents that are known to be covered by all the data sources under analysis, and then they compare the list of citing documents that each data source is able to find for each of the seed documents (Martín-Martín et al. 2018). The rationale of this method is that if data source $A$ is not able to find a citation that data source $B$ has found, the reason must be that

\footnotetext{
${ }^{8}$ In the case of COCI, the results cannot reflect the full coverage of CrossRef given the incomplete availability of reference lists in this source. Nevertheless, including it in the analysis will inform us of what proportion of citations are currently available in the public domain.
} 
the citing document is not covered by data source $A$. This assumes that all data sources are equally effective in detecting citation relationships. In fact, each data source has its own (usually secret) citation detection algorithms, and small discrepancies in citation data across databases exist even when removing the factor of differences in coverage (van Eck and Waltman 2019; Visser et al. 2020). Furthermore, it is known that bibliographic databases do not always have access to cited reference lists for all the documents they cover, which also affects the citations they can detect. For example, reference lists are only available in a fraction of the documents indexed in CrossRef, so an analysis of the citations detected in this source does not accurately reflect the true size of the bibliographic database. Other sources, especially academic search engines, are also affected by this issue to some degree. ${ }^{9}$ Lastly, academic documents that do not cite and are not cited by other documents cannot be detected by this type of analysis. Therefore, results from studies that analyse citations to identify relative differences in the sizes of bibliographic databases are likely to be affected by these confounding factors.

Of the six data sources that are analysed in this study, only two (Microsoft Academic and $\mathrm{COCI}$ ) offered free and unrestricted access to the complete list of documents (or citation relationships in the case of COCI) that they covered at the time of data collection, although Dimensions now also offers this to researchers. To include all data sources in this study in a comparable way, the alternative method (selection of seed sample and analysis of citations) was used to discover relative coverage differences among data sources across subject categories. Since citation extraction discrepancies seem likely to be small compared to coverage differences, the results should also be useful to detect differences in coverage between sources.

\section{Selection of seed sample}

The sample of citations analysed in this paper was taken from a seed sample of highlycited documents: those listed in Google Scholar's Classic Papers product ${ }^{10}$ (GSCP). This sample comprises the top 10 most cited documents published in 2006 according to Google Scholar in each of 252 subject categories (except French Studies, which has only 5 documents). The 252 subject categories are also assigned to one or more of 8 broad subject areas. The seed sample contains a total of 2515 highly-cited documents. For more information on GSCP, see Orduna-Malea et al. (2018).

This seed sample was considered useful for the purpose of this study, as it is the only sample of documents in Google Scholar for which an article-level subject classification is available. At the time of data collection, no other sample of documents with an articlelevel classification was readily available to us, and a sample with these characteristics was considered superior to the journal-level classification schemes that are used in sources such as Web of Science and Scopus. Additionally, being aware of the difficulties that extracting data from Google Scholar entail (Else 2018), the election of a sample of documents that were known to be highly cited also guaranteed a high efficiency in the citation extraction

\footnotetext{
9 Visser et al. (2020) found that a large number of citations missing from Microsoft Academic were caused by missing reference lists in the citing documents. As far as we know no study has analysed how many missing citations in Google Scholar are caused by missing reference lists.

$10 \mathrm{https} / / /$ scholar.google.com/citations?view_op=list_classic_articles\&hl=en\&by=2006.
} 
process (each request to Google Scholar retrieved the maximum amount of records that the search engine displays per page).

This study analyses the complete list of documents that cite this seed sample, as reported in a variety of citation indexes (Google Scholar, Microsoft Academic, Scopus, Dimensions, Web of Science, and COCI). In this study, they are called citing documents, or more simply, citations. Thus, this study follows the same approach as Martín-Martín et al. (2018).

\section{Collection of citation data}

Each of the 2515 highly-cited documents were searched on Google Scholar, Microsoft Academic, Scopus, Dimensions, WoS, and COCI (Table 1). For each seed document found in a data source, the list of citing documents was extracted, as described below. The searches and data extraction were carried out in May and June 2019 (i.e., not re-using the data from the previous paper).

Google Scholar has no data exporting capabilities in its web interface and no API. Instead, a custom web scraper was used to extract the list of citing documents for each highly-cited document in the seed sample (Martín-Martín 2018). CAPTCHAs were solved manually when they appeared.

Google Scholar provides up to 1000 results per query. In order to download the complete list of citing documents for those with more than 1000 citations, queries were split by the publication year of the citing documents. Using this method, we were able to download most of the citing documents available in Google Scholar: for 2429 (96.5\%) seed documents, we were able to extract a list of citing documents, amounting to at least $98 \%$ of the total citation counts reported by Google Scholar for these seed documents. In eight cases (extremely highly-cited seed documents), splitting queries by publication year was not enough to find all possible citing documents, and in these cases the number of citing documents extracted from Google Scholar was lower than $75 \%$ of the reported Google Scholar citation counts. This disadvantages Google Scholar in comparison to the other sources, for which all citing documents could be extracted. 2,689,809 citations were extracted from Google Scholar.

The metadata provided by Google Scholar is limited (Delgado López-Cózar et al. 2019). For example, Google Scholar does not provide the DOI of a document, which is very useful for document matching across data sources, and therefore relevant to our study. To enrich the limited metadata provided by Google Scholar, we followed several approaches. First, given that most of the citing documents from Google Scholar had already been analysed (Martín-Martín et al. 2018), we matched the newly extracted list of citing documents to the data from the previous study, and retrieved all the enriched metadata that was available in the dataset used for the 2018 study. Next, for all the citing documents that could not be matched in the previous step (mostly newer citations), metadata was extracted from the HTML Meta tags in the landing page of each citing document, and with public metadata APIs when a CrossRef or DataCite DOI could be found. These methods produced a DOI for $62.9 \%$ of all Google Scholar citations.

To collect citation data from Microsoft Academic, the Academic Search API ${ }^{11}$ was used. This API is free with a limit of 10,000 transactions per month. At the moment of data collection, this API did not facilitate searching directly by DOI (Thelwall 2018b). For this

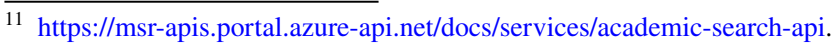


Table 1 No. of seed highly-cited documents and citations found in each data source

\begin{tabular}{llll}
\hline Source & \multicolumn{2}{l}{ Seed documents } & Citations \\
\cline { 2 - 3 } & $N$ & $\%$ & \\
\hline Google Scholar & 2515 & 100 & $2,689,809$ \\
Microsoft Academic & 2500 & 99.4 & 1840,702 \\
Scopus & 2447 & 97.3 & $1,738,573$ \\
Dimensions & 2478 & 98.5 & $1,649,162$ \\
WoS & 2342 & 93.1 & $1,503,657$ \\
COCI & 2471 & 98.3 & 852,413 \\
\hline
\end{tabular}

${ }^{\text {a}}$ Due to the sample selection process, the figures related to the seed documents found in each data source cannot be used as evidence that Google Scholar has higher coverage than the other sources

reason, every highly-cited seed document was first searched for by title. Once the seed document was retrieved and confirmed to be correct, new queries were submitted to retrieve the list of citing documents. Up to 1000 citing documents per query could be extracted (seed documents with over 1000 citations required more than one query to extract all citations). For each citing document, the Microsoft Academic internal Id, as well as the DOI, the document title, the list of authors, the publication year, the language, and the citation counts, were retrieved. 1,840,702 citations were extracted from Microsoft Academic.

To collect citation data from Scopus, the exporting capabilities of the web interface were used. Each seed highly-cited document was searched in Scopus by DOI and title, and, if found, the list of citing documents was exported in csv format. Scopus allows 2000 records per query to be exported. When seed documents had over 2000 citations, the alternative email service was used, which allows 20,000 records to be exported. No document in the seed sample had more than 20,000 citations in Scopus. 1,738,573 citations were extracted from Scopus.

To collect citation data from Dimensions, its API was used, which is free for research. ${ }^{12}$ The Dimensions API allows searching by DOI. Therefore, all seed highly-cited documents were searched for using their DOI, and, when unavailable, by their title. Once all the seed documents had been identified in Dimensions, the API was also used to extract the list of citing documents. For each citation, the basic bibliographic information (DOI, title, authors, publication year, source, document type) was recorded. 1,649,162 citations were extracted from Dimensions.

To collect citation data from WoS, the web interface was used. All citation indexes in WoS Core Collection were included in the analysis, including the Emerging Sources Citation Index (from publication year 2005 to the present). Each seed highly-cited document was searched by its DOI, and, when unavailable, by its title. The list of citing documents was then exported in batches of up to 500. The exported files were consolidated into a single table using a set of R functions (Martín-Martín and Delgado López-Cózar 2016). 1,503,657 citations were extracted from WoS.

To collect citation data from COCI, the public API was used. The DOI of each seed highly-cited document was searched in order to retrieve the complete list of citing DOIs. 852,413 citation relationships were extracted from COCI.

12 https://www.dimensions.ai/scientometric-research/. 


\section{Analysis of citation data}

To calculate citation overlaps across data sources, the citing documents from different data sources were matched. The matching process started with two data sources (WoS and Scopus), and the result was a full join of the two sources: a table containing all citations found both by WoS and Scopus, as well as the citations found only by one of the data sources. The resulting dataset was matched to the data obtained from another data source (Dimensions), and this process was repeated until all data sources were merged into a master list of citations (Table 2). The matching criteria are below, and are the same as previously used (Martín-Martín et al. 2018):

1. For each pair of data sources $A$ and $B$ and a seed highly-cited document $X$, all citing documents with a DOI that cite $X$ according to $A$ where matched to all citing documents with a DOI that cite $X$ according to $B$.

2. For each of the unmatched documents citing $X$ in $A$ and $B$, a further comparison was carried out (except in the matching round where COCI data was integrated into the master table). The title of each unmatched document citing $X$ in $A$ was compared to the titles of all the unmatched documents citing $X$ in $B$, using the restricted Damerau-Levenshtein distance (optimal string alignment) (Damerau 1964; Levenshtein 1966). The pair of citing documents which returned the highest title similarity ( 1 is perfect similarity) was selected as a potential match. This match was considered successful if either of the following conservative heuristics was met:

- The title similarity was at least 0.8 , and the title of the citing document was at least 30 characters long (to avoid matches between short, undescriptive titles such as "Introduction").

- The title similarity was at least 0.7 , and the first author of the citing document was the same in $A$ and $B$.

The matching criteria described above are intentionally conservative, so a match is only accepted when the two documents have very similar metadata. The analysis does not attempt to remove duplicate citations within the same data source, although Google Scholar and Scopus (and perhaps others) are afflicted by this issue (Orduna-Malea et al. 2017; van Eck and Waltman 2019). In this study, if there are duplicate citations within the same data source only one of the instances will be linked to the same citation in other sources, while the rest will (erroneously) appear as unique citations. Therefore, the percentage overlaps between sources calculated are conservative estimates (i.e., they might be higher than reported here). A replication of the overlap analysis carried in Martín-Martín et al. (2018) for one subject category (Operations Management) showed that overlap figures are affected little when duplicates are identified and removed, however (Chapman and Ellinger 2019).

Given that the objective is to detect relative differences in coverage across databases, to make comparisons as fair as possible the subset of citations that are considered in each comparison is adapted to include only citation relationships where the cited seed document is covered by all sources present in the comparison. For example, in a comparison of coverage across the six data sources analysed in this study (Table 1, top), only citations 
Table 2 Rounds of the matching process

\begin{tabular}{llll}
\hline Matching round & Data sources being matched & Resulting dataset & Merged citations \\
\hline 1st & WoS $\bowtie$ Scopus & master_1 & $1,852,681$ \\
2nd & master_1 $\bowtie$ Dimensions & master_2 & $1,990,862$ \\
3rd & master_2 $\bowtie$ Microsoft Academic & master_3 & $2,263,896$ \\
4th & master_3 $\bowtie$ COCI & master_4 & $2,273,067$ \\
5th & master_4 $\bowtie$ Google Scholar & master_5 & $3,073,351$ \\
\hline
\end{tabular}

to the 2319 seed highly-cited documents covered by all six data sources are considered. However, in pairwise comparisons, such as the Venn diagram that represents overlapping and unique citations in Google Scholar and Microsoft Academic (Fig. 2a), the citations to the 2500 seed highly-cited documents that are known to be covered by these two sources were analysed.

Data processing was carried out with the R programming language (R Core Team 2014) using several R packages and custom functions (Dowle et al. 2018; Krassowski 2020; Larsson et al. 2018; Martín-Martín and Delgado López-Cózar 2016; van der Loo et al. 2018; Walker and Braglia 2018; Wickham 2016; Wilke 2019). The resulting data files are openly available. $^{13}$

\section{Results}

\section{Overall results (all subject categories)}

\section{Relative overlap}

Overall, Google Scholar has the highest coverage, as it found $88 \%$ of all possible citations $(2,918,105)$ to the 2319 highly-cited documents in our sample that were covered by the six sources under analysis (Fig. 1, first row). Microsoft Academic, Scopus, Dimensions and WoS found substantially fewer (60-52\% of all citations). COCI found only $28 \%$ of all possible citations.

In terms of relative overlaps between two data sources, larger data sources are able to find a vast majority of the citations found by the smaller data sources (Fig. 1, row 2 through 6). Thus, Google Scholar found $89 \%$ of the citations in the second data source with the largest coverage (Microsoft Academic), and up to $94 \%$ of the citations in the smaller sources (WoS, COCI). On the other side of the spectrum, COCI, the smallest source, found between $30 \%$ and $51 \%$ of the citations found by the other sources (Google Scholar and Dimensions, respectively).

For Microsoft Academic, Scopus, Dimensions, and WoS, the relative overlap between any two of these sources ranges from high (WoS found $73 \%$ of the citations

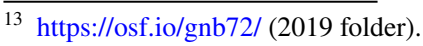




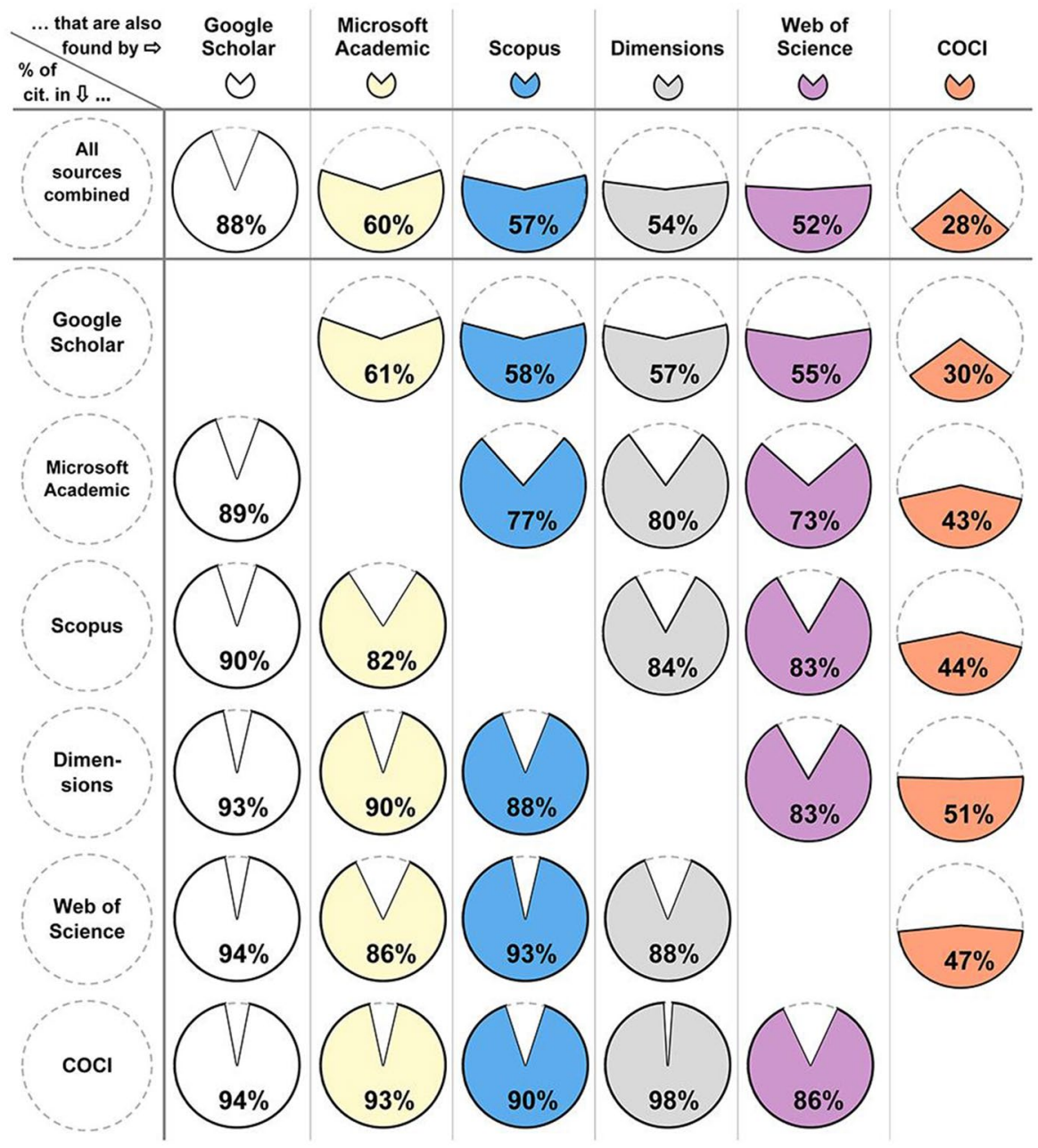

Fig. 1 Percentage of citations found by each database, relative to all citations (first row), and relative to citations found by the other databases (subsequent rows)

found by Microsoft Academic) to almost full overlap (Dimensions found $98 \%$ of the citations available in COCI). Figure 1 shows that it is not always the case that the larger the source, the higher the proportion of citations from another source that it will be able to find. For example, Dimensions found $80 \%$ of the citations available in Microsoft Academic, while Scopus (larger than Dimensions) found 77\%. The cause of this might be that both Microsoft Academic and Dimensions cover non-journal content, such as 
preprints, while Scopus does not. Scopus found 93\% of the citations found by WoS, while Microsoft Academic (larger than Scopus) found 86\%. Dimensions was able to find the highest proportion of COCI citations (98\%) out of all the other sources (including Google Scholar).

\section{Overlaps within the full set of citations}

A quarter (26\%) of all citations were found only by Google Scholar (Fig. 2), 21\% of the citations were found by the six sources, while $18 \%$ were found by all sources except COCI. The remaining $35 \%$ were found by combinations of four or less data sources, and the highest values were found in sectors that include Google Scholar and/or Microsoft Academic.

When considering all possible pairwise combinations (Fig. 3), the pairs of data sources that are most similar in terms of full citation overlap are Scopus/WoS (78\% of all citations found by either were found by both), followed by Scopus/Dimensions (75\%), Dimensions/WoS (75\%), and Microsoft Academic/Dimensions (74\%). Pairs that include Google Scholar or COCI display lower percentages of overlap: in the case of Google Scholar this is caused by the extra coverage in Google Scholar that is not found in the other sources, while for $\mathrm{COCI}$ the reason is the opposite.

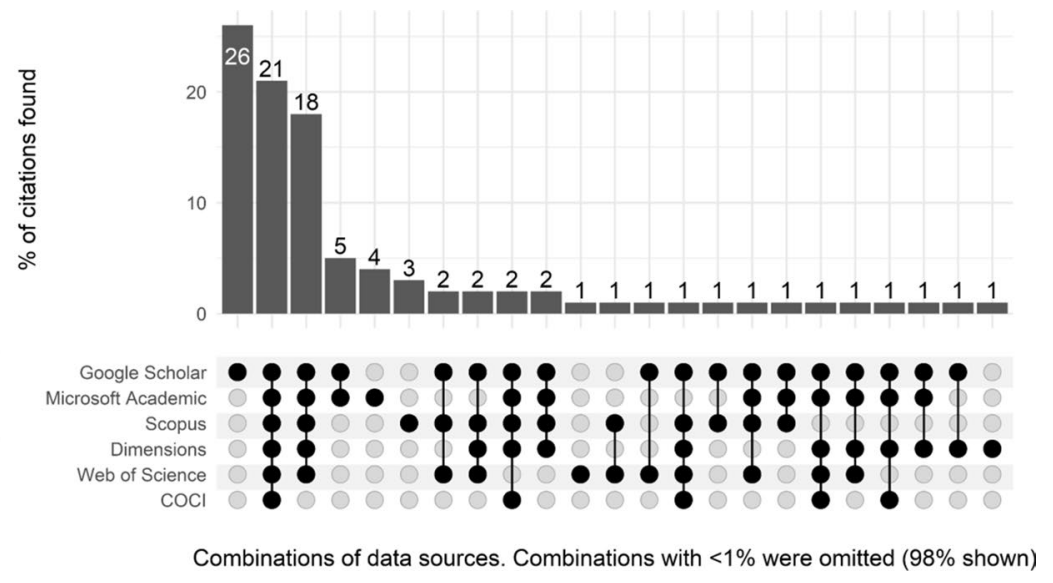

Fig. 2 Overlaps of citations found by Google Scholar, Microsoft Academic, Scopus, Dimensions, Web of Science, and COCI. Values expressed as percentages relative to $N=2,918,105$ citations to 2319 documents. Combinations with values below $1 \%$ are not displayed 


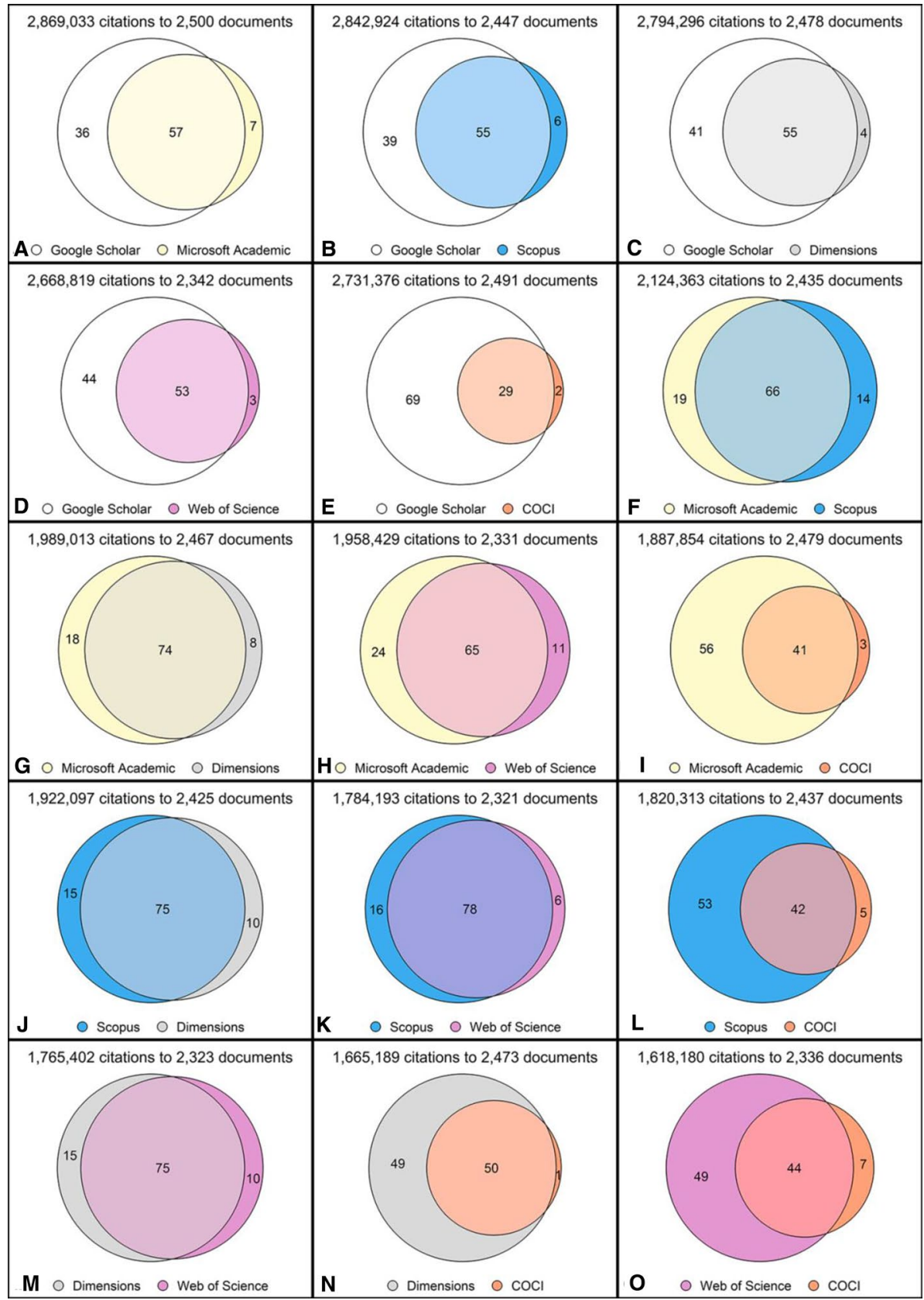

Fig. 3 Comparison of citing document overlaps between Google Scholar, Microsoft Academic, Scopus, Dimensions, Web of Science, and COCI (pairwise). Figures within Venn diagrams expressed as percentages 


\section{Analysis by subject areas and categories}

\section{Relative overlap}

Disaggregating the data by broad subject areas provides a more detailed picture of the coverage of these sources. Google Scholar found the great majority of citations (85-90\%) in all eight subject areas (Table 3) and COCI found the fewest. COCI has differences in coverage across areas: in the Humanities and Social Sciences it found $18-20 \%$ of all citations, while in the STEM areas (Science, Technology, Engineering, and Mathematics) it found a higher proportion of citations (27-32\%).

Between these two extremes, the other four sources (Microsoft Academic, Scopus, Dimensions, and WoS) tend to have similar coverage of each field, but differences between fields (Table 3). They have more comprehensive coverage for Chemical \& Material Sciences (69-72\%), followed by Life Sciences \& Earth Sciences (60-68\%). Conversely, their coverage is much lower in Humanities, Literature \& Arts (25-39\%), Social Sciences (33-47\%) and Business, Economics \& Management (29-47\%). Among these four, Microsoft Academic seems to have the most comprehensive coverage, except in Physics \& Mathematics, where it found fewer of the citations (57\%) than the other sources.

Further disaggregating the data to identify the percentage of relative citation overlap for each pair of sources in each subject area (Table 4), the patterns for the complete dataset (Fig. 1) recur. Google Scholar consistently found most citations found by the other sources across all areas; there is a higher relative overlap between Microsoft Academic and Dimensions/COCI than between Microsoft Academic and Scopus/WoS; conversely, the relative overlap between Scopus and WoS is always higher than between Scopus and other sources; the highest relative overlap in each area is always for Dimensions/COCI; Microsoft Academic seems to lack coverage in Physics \& Mathematics, as evidenced by its lower relative overlap in this area.

Table 3 Percentage of citations found by each data source, relative to the total number of citations found overall and by broad areas

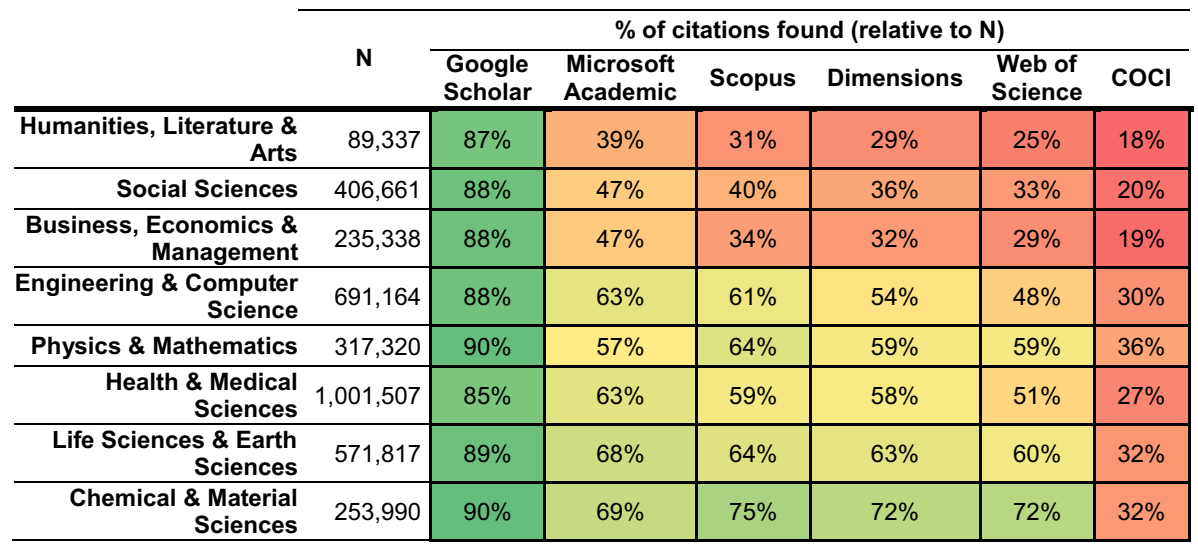


Table 4 Relative pairwise overlaps between data sources (\%). Overall and by broad subject areas

A Humanities, Literature \& Arts

\begin{tabular}{|r|c|c|c|c|c|c|}
\hline $\begin{array}{c}\ldots \\
\text { that are also } \\
\text { found by } \Rightarrow \\
\text { Percentage } \\
\text { of citations in \... }\end{array}$ & $\begin{array}{c}\text { Google } \\
\text { Scholar }\end{array}$ & $\begin{array}{c}\text { Microsoft } \\
\text { Academic }\end{array}$ & Scopus & Dimensions & $\begin{array}{c}\text { Web of } \\
\text { Science }\end{array}$ & COCl \\
\hline Google Scholar & & $39 \%$ & $33 \%$ & $30 \%$ & $29 \%$ & $19 \%$ \\
\hline Microsoft Acad. & $86 \%$ & & $57 \%$ & $62 \%$ & $53 \%$ & $42 \%$ \\
\hline Scopus & $84 \%$ & $68 \%$ & & $65 \%$ & $68 \%$ & $42 \%$ \\
\hline Dimensions & $89 \%$ & $86 \%$ & $75 \%$ & & $69 \%$ & $59 \%$ \\
\hline Web of Science & $87 \%$ & $73 \%$ & $80 \%$ & $70 \%$ & & $46 \%$ \\
\hline COCl & $93 \%$ & $92 \%$ & $77 \%$ & $94 \%$ & $73 \%$ & \\
\hline
\end{tabular}

\section{B Social Sciences}

\begin{tabular}{|r|c|c|c|c|c|c|}
\hline $\begin{array}{r}\ldots \text { that are also } \\
\text { found by } \Rightarrow \\
\begin{array}{c}\text { Percentage } \\
\text { of citations in \ु... }\end{array}\end{array}$ & $\begin{array}{c}\text { Google } \\
\text { Scholar }\end{array}$ & $\begin{array}{c}\text { Microsoft } \\
\text { Academic }\end{array}$ & Scopus & Dimensions & $\begin{array}{c}\text { Web of } \\
\text { Science }\end{array}$ & COCI \\
\hline Google Scholar & & $48 \%$ & $41 \%$ & $39 \%$ & $37 \%$ & $22 \%$ \\
\hline Microsoft Acad. & $88 \%$ & & $66 \%$ & $69 \%$ & $60 \%$ & $40 \%$ \\
\hline Scopus & $89 \%$ & $78 \%$ & & $75 \%$ & $76 \%$ & $43 \%$ \\
\hline Dimensions & $93 \%$ & $90 \%$ & $83 \%$ & & $76 \%$ & $54 \%$ \\
\hline Web of Science & $92 \%$ & $82 \%$ & $88 \%$ & $81 \%$ & & $47 \%$ \\
\hline COCl & $96 \%$ & $95 \%$ & $85 \%$ & $96 \%$ & $80 \%$ & \\
\hline
\end{tabular}

\section{Business, Economics \& Management}

\begin{tabular}{|c|c|c|c|c|c|c|}
\hline $\begin{array}{l}\text {.... that are also } \\
\text { found by } \Rightarrow \\
\begin{array}{l}\text { Percentage } \\
\text { of citations in } \sqrt{3} . . .\end{array}\end{array}$ & $\begin{array}{l}\text { Google } \\
\text { Scholar }\end{array}$ & $\begin{array}{l}\text { Microsoft } \\
\text { Academic }\end{array}$ & Scopus & Dimensions & $\begin{array}{l}\text { Web of } \\
\text { Science }\end{array}$ & $\mathrm{COCl}$ \\
\hline Google Scholar & & $46 \%$ & $35 \%$ & $34 \%$ & $31 \%$ & $20 \%$ \\
\hline Microsoft Acad. & $85 \%$ & & $58 \%$ & $61 \%$ & $52 \%$ & $36 \%$ \\
\hline Scopus & $91 \%$ & $80 \%$ & & $77 \%$ & $75 \%$ & $45 \%$ \\
\hline Dimensions & $93 \%$ & $90 \%$ & $82 \%$ & & $75 \%$ & $55 \%$ \\
\hline Web of Science & $93 \%$ & $84 \%$ & $87 \%$ & $83 \%$ & & $50 \%$ \\
\hline $\mathrm{COCl}$ & $94 \%$ & $92 \%$ & $83 \%$ & $95 \%$ & $78 \%$ & \\
\hline
\end{tabular}

\section{Engineering \& Computer Science}

\begin{tabular}{|c|c|c|c|c|c|c|}
\hline $\begin{array}{l}\text {.... that are also } \\
\text { found by } \Rightarrow \\
\text { Percentage } \\
\text { of citations in } \sqrt{1} . . .\end{array}$ & $\begin{array}{l}\text { Google } \\
\text { Scholar }\end{array}$ & $\begin{array}{r}\text { Microsoft } \\
\text { Academic }\end{array}$ & Scopus & Dimensions & $\begin{array}{l}\text { Web of } \\
\text { Science }\end{array}$ & $\mathrm{COCl}$ \\
\hline Google Scholar & & $65 \%$ & $62 \%$ & $58 \%$ & $55 \%$ & $32 \%$ \\
\hline Microsoft Acad. & $90 \%$ & & $79 \%$ & $78 \%$ & $70 \%$ & $43 \%$ \\
\hline Scopus & $89 \%$ & $82 \%$ & & $81 \%$ & $79 \%$ & $45 \%$ \\
\hline Dimensions & $93 \%$ & $91 \%$ & $91 \%$ & & $82 \%$ & $53 \%$ \\
\hline Web of Science & $93 \%$ & $86 \%$ & $94 \%$ & $87 \%$ & & $49 \%$ \\
\hline $\mathrm{COCl}$ & $94 \%$ & $94 \%$ & $92 \%$ & $97 \%$ & $83 \%$ & \\
\hline
\end{tabular}

\section{E Physics \& Mathematics}

\begin{tabular}{|c|c|c|c|c|c|c|}
\hline $\begin{array}{l}\text {.... that are also } \\
\text { found by } \Rightarrow \\
\text { Percentage } \\
\text { of citations in } \sqrt{3} \ldots\end{array}$ & $\begin{array}{l}\text { Google } \\
\text { Scholar }\end{array}$ & $\begin{array}{l}\text { Microsoft } \\
\text { Academic }\end{array}$ & Scopus & Dimensions & $\begin{array}{l}\text { Web of } \\
\text { Science }\end{array}$ & $\mathrm{COCl}$ \\
\hline Google Scholar & & $58 \%$ & $65 \%$ & $61 \%$ & $61 \%$ & $37 \%$ \\
\hline Microsoft Acad. & $91 \%$ & & $83 \%$ & $83 \%$ & $78 \%$ & $48 \%$ \\
\hline Scopus & $91 \%$ & $74 \%$ & & $85 \%$ & $87 \%$ & $52 \%$ \\
\hline Dimensions & $93 \%$ & $80 \%$ & $93 \%$ & & $88 \%$ & $60 \%$ \\
\hline Web of Science & $93 \%$ & $75 \%$ & $95 \%$ & $88 \%$ & & $55 \%$ \\
\hline $\mathrm{COCl}$ & $92 \%$ & $77 \%$ & $94 \%$ & $98 \%$ & $90 \%$ & \\
\hline
\end{tabular}


Table 4 (continued)

F Health \& Medical Sciences

\begin{tabular}{|c|c|c|c|c|c|c|}
\hline $\begin{array}{l}\text {.... that are also } \\
\text { found by } \Rightarrow \\
\text { Percentage } \\
\text { of citations in } \sqrt{3} . . .\end{array}$ & $\begin{array}{l}\text { Google } \\
\text { Scholar }\end{array}$ & $\begin{array}{l}\text { Microsoft } \\
\text { Academic }\end{array}$ & Scopus & Dimensions & $\begin{array}{l}\text { Web of } \\
\text { Science }\end{array}$ & $\mathrm{COCl}$ \\
\hline Google Scholar & & $64 \%$ & $61 \%$ & $62 \%$ & $58 \%$ & $29 \%$ \\
\hline Microsoft Acad. & $87 \%$ & & $78 \%$ & $84 \%$ & $75 \%$ & $41 \%$ \\
\hline Scopus & $88 \%$ & $84 \%$ & & $86 \%$ & $84 \%$ & $40 \%$ \\
\hline Dimensions & $91 \%$ & $91 \%$ & $86 \%$ & & $82 \%$ & $45 \%$ \\
\hline Web of Science & $95 \%$ & $87 \%$ & $92 \%$ & $89 \%$ & & $43 \%$ \\
\hline $\mathrm{COCl}$ & $94 \%$ & $96 \%$ & $89 \%$ & $99 \%$ & $86 \%$ & \\
\hline
\end{tabular}

\section{G Life Sciences \& Earth Sciences}

\begin{tabular}{|r|c|c|c|c|c|c|}
\hline $\begin{array}{r}\ldots \\
\text { that are also } \\
\text { found by } \Rightarrow \\
\text { Percentage } \\
\text { of citations in \... }\end{array}$ & $\begin{array}{c}\text { Google } \\
\text { Scholar }\end{array}$ & $\begin{array}{c}\text { Microsoft } \\
\text { Academic }\end{array}$ & Scopus & Dimensions & $\begin{array}{c}\text { Web of } \\
\text { Science }\end{array}$ & COCl \\
\hline Google Scholar & & $69 \%$ & $67 \%$ & $67 \%$ & $64 \%$ & $34 \%$ \\
\hline Microsoft Acad. & $91 \%$ & & $82 \%$ & $86 \%$ & $80 \%$ & $45 \%$ \\
\hline Scopus & $93 \%$ & $88 \%$ & & $88 \%$ & $88 \%$ & $46 \%$ \\
\hline Dimensions & $94 \%$ & $93 \%$ & $90 \%$ & & $87 \%$ & $50 \%$ \\
\hline Web of Science & $95 \%$ & $91 \%$ & $94 \%$ & $91 \%$ & & $48 \%$ \\
\hline COCl & $96 \%$ & $96 \%$ & $92 \%$ & $98 \%$ & $90 \%$ & \\
\hline
\end{tabular}

\section{H Chemical \& Material Sciences}

\begin{tabular}{|r|c|c|c|c|c|c|}
\hline $\begin{array}{c}\ldots \text { that are also } \\
\text { found by } \Rightarrow \\
\begin{array}{c}\text { Percentage } \\
\text { of citations in \... }\end{array}\end{array}$ & $\begin{array}{c}\text { Google } \\
\text { Scholar }\end{array}$ & $\begin{array}{c}\text { Microsoft } \\
\text { Academic }\end{array}$ & Scopus & Dimensions & $\begin{array}{c}\text { Web of } \\
\text { Science }\end{array}$ & Cocl \\
\hline Google Scholar & & $71 \%$ & $78 \%$ & $75 \%$ & $75 \%$ & $34 \%$ \\
\hline Microsoft Acad. & $93 \%$ & & $90 \%$ & $92 \%$ & $88 \%$ & $43 \%$ \\
\hline Scopus & $93 \%$ & $83 \%$ & & $89 \%$ & $92 \%$ & $40 \%$ \\
\hline Dimensions & $94 \%$ & $89 \%$ & $94 \%$ & & $91 \%$ & $44 \%$ \\
\hline Web of Science & $94 \%$ & $84 \%$ & $96 \%$ & $90 \%$ & & $41 \%$ \\
\hline COCl & $95 \%$ & $93 \%$ & $93 \%$ & $98 \%$ & $91 \%$ & \\
\hline
\end{tabular}

\section{Full overlap}

The differences in coverage between the older (Google Scholar, Scopus, WoS) and newer (Microsoft Academic, Dimensions) sources across subject areas are also evident from three-way comparisons (Figs. 4, 6, 8). The three-set combinations of data sources that are not displayed here are accessible from "Appendix 1". The combinations that include more than one of the older sources are not included here because they were discussed in a previous study (Martín-Martín et al. 2018) and the results have barely changed. The combinations that include COCI are not displayed here because it is essentially a subset of the other sources (especially Dimensions).

Venn diagrams for the 252 specific subject categories are also accessible from "Appendix 1". Figures 5, 7, 9 and 10 display the distribution of the proportions of citations that would fall in each section of the Venn diagrams calculated at this level of aggregation, for various pairs of data sources. The remaining combinations are accessible from "Appendix 2".

\section{Google Scholar and the new sources: Microsoft Academic, and Dimensions}

For Google Scholar, Microsoft Academic, and Dimensions, the largest percentages of full overlap (citations found by the three sources) occur in the STEM fields (Fig. 4). These 
Google Scholar $\bigcirc$ Microsoft Academic Dimensions

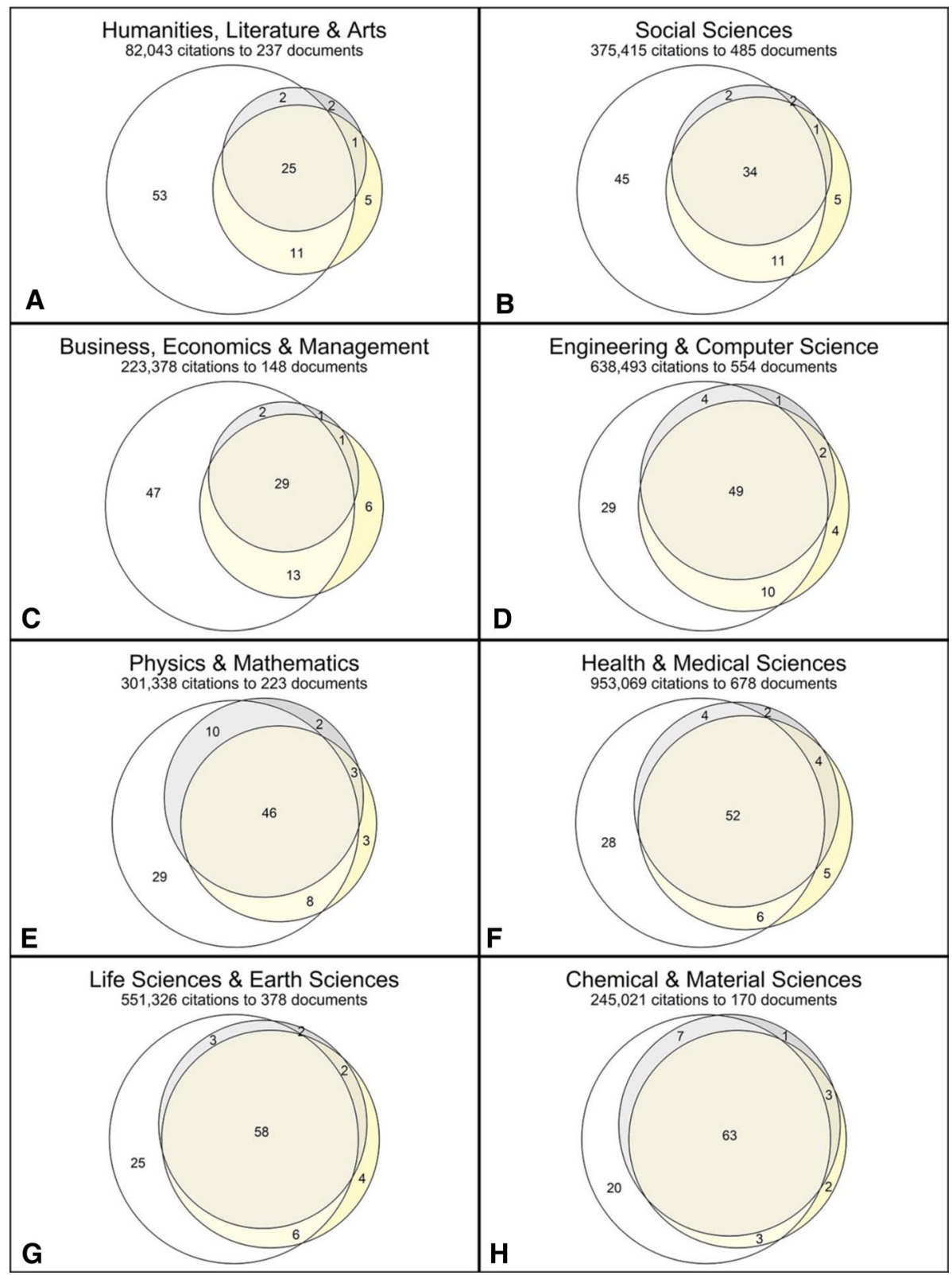

Fig. 4 Overlaps between citations found by Google Scholar, Microsoft Academic, and Dimensions in broad subject areas. Figures within Venn diagrams expressed as percentages

range from $46 \%$ in Physics and Mathematics, to 63\% in Chemical and Material Sciences. Full overlap in the areas of Humanities and Social Sciences is distinctly lower $(25-34 \%)$. This is caused by lower coverage of these areas in Microsoft Academic and Dimensions. 


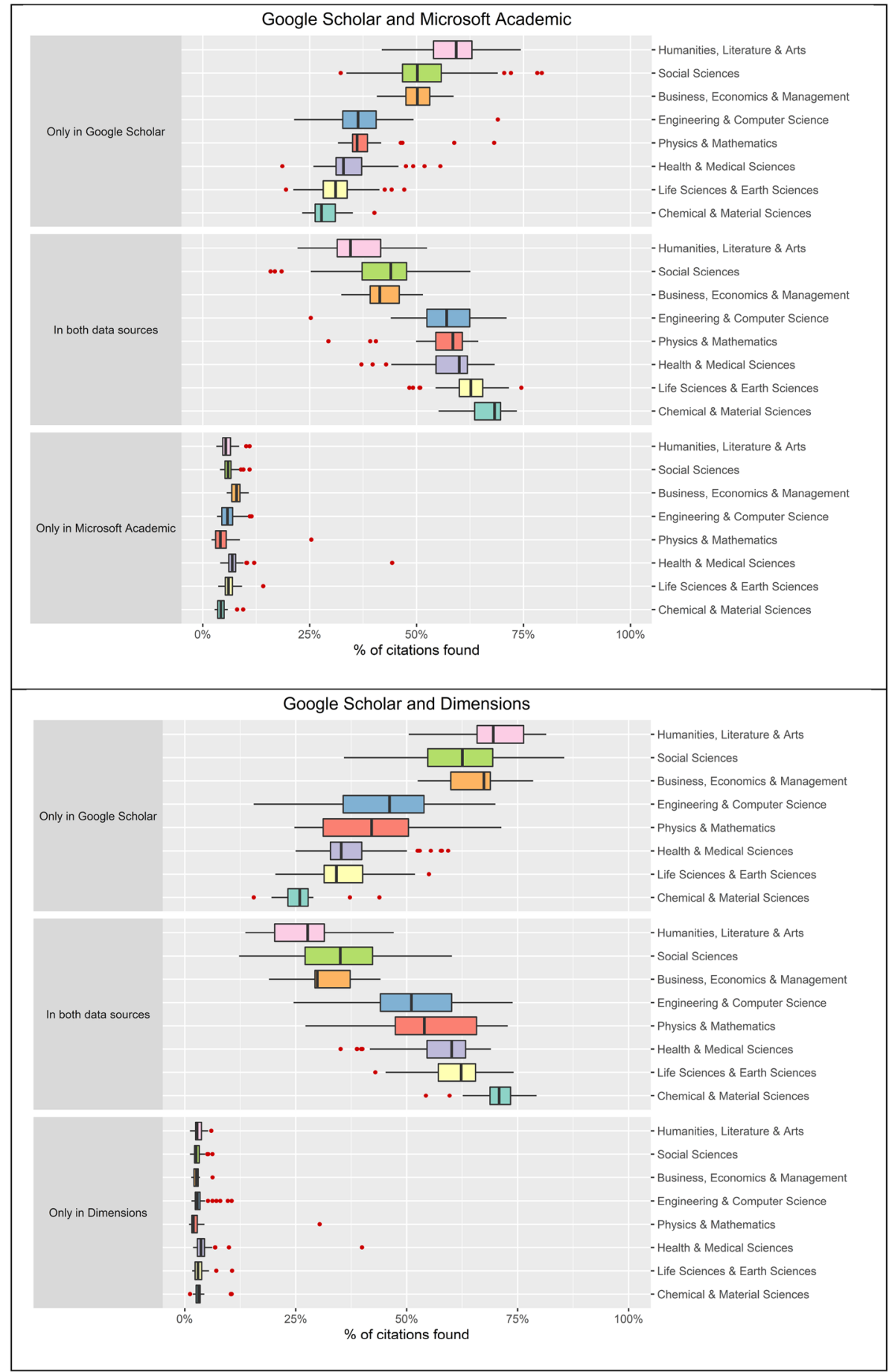

Fig. 5 Distribution of citations that fall within each sector of the Venn diagrams that compare Google Scholar to Microsoft Academic and Dimensions. Calculated at the level of subject categories, and aggregated by subject areas 
The percentage of citations in Microsoft Academic and/or Dimensions that is not covered by Google Scholar ranges from 6\% (in Chemical and Material Sciences) to 11\% (in Health \& Medical Sciences).

At the level of specific subject categories, for pairwise comparisons between Google Scholar/Microsoft Academic and Google Scholar/Dimensions (Fig. 5) the general trend of the subject area is followed, with variations in some subject categories. The variation seems to be higher between Google Scholar/Dimensions than between Google Scholar/ Microsoft Academic. Nevertheless, in both comparisons the percentages in the sector "Only in Google Scholar" are higher in the Humanities and Social Sciences, and lower in STEM fields. The sector "In both data sources" almost mirrors the one above, and the sectors "Only in Microsoft Academic" and "Only in Dimensions" have values almost exclusively below $10 \%$, with two major exceptions. These correspond to the categories Astronomy \& Astrophysics, ${ }^{14}$ and Psychology. ${ }^{15}$ In these two categories, many citations found by Microsoft Academic and Dimensions were not found by Google Scholar. In the case of Psychology, the low citation coverage in Google Scholar is caused by one extremely highly-cited document (Using thematic analysis in psychology, by Virginia Braun and Victoria Clarke ${ }^{16}$ ), which at the time of data collection had 54,323 citations in Google Scholar. However, because of the limitations of Google Scholar's search interface for data extraction, only 10,996 citations could be extracted from Google Scholar for this article.

\section{Scopus and the new sources: Microsoft Academic and Dimensions}

For Microsoft Academic, Scopus, and Dimensions, none of the sources is always larger than the others, with the results varying by subject area (Fig. 6). Microsoft Academic sometimes has larger coverage than Scopus (Humanities and Social Sciences), although in these areas both contribute many unique citations. Scopus also sometimes provides more coverage than Microsoft Academic (Physics \& Mathematics, Chemical \& Material Sciences). The previously seen trend of higher percentages of full overlap in STEM fields also occurs here. The number of citations found by Dimensions is similar to that of Scopus across all subject areas, but there are also many citations that one of them finds that the other does not in the Humanities and Social Sciences. Comparing the three sources together, Dimensions provides the fewest unique citations.

In most subject categories (Fig. 7), there are large Microsoft Academic/Scopus and Scopus/Dimensions citation overlaps. This is especially evident in STEM categories, where

\footnotetext{
${ }^{14}$ Google Scholar/Microsoft Academic: https://osf.io/g8z42/; Google Scholar/Dimensions: https://osf.io/ bwv5s/.

15 Google Scholar/Microsoft Academic: https://osf.io/jqwah/; Google Scholar/Dimensions: https://osf.io/ xnf24/.

16 https://www.tandfonline.com/doi/abs/10.1191/1478088706QP063OA.
} 
Scopus

Microsoft Academic Dimensions

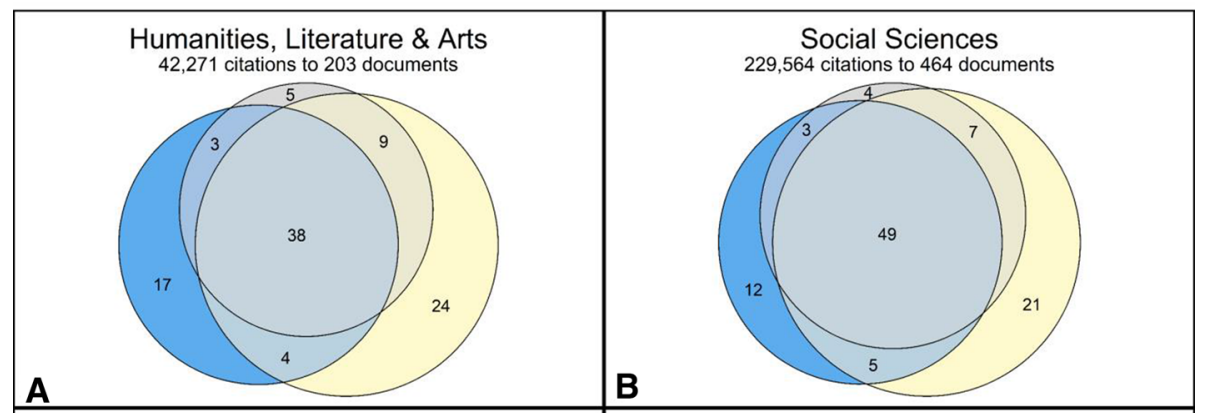

Business, Economics \& Management 129,493 citations to 145 documents

C

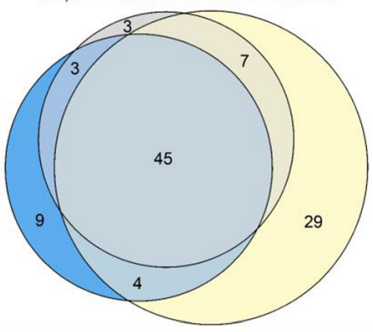

Physics \& Mathematics

236,010 citations to 222 documents

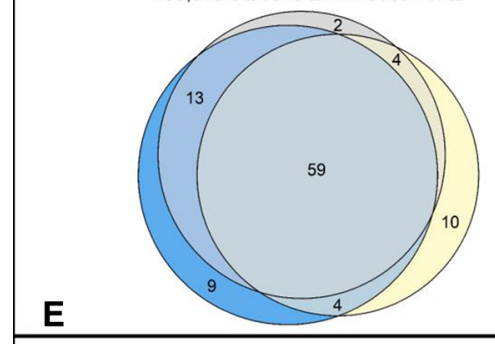

Life Sciences \& Earth Sciences

442,250 citations to 377 documents

G

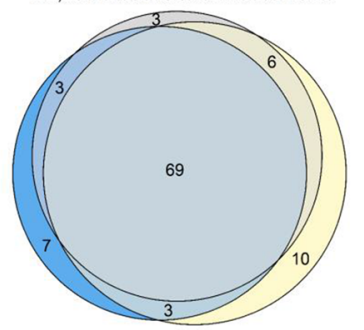

D

Engineering \& Computer Science

501,231 citations to 549 documents

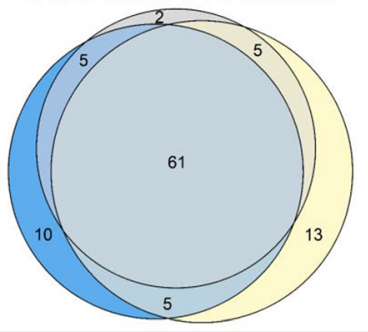

Health \& Medical Sciences

748,401 citations to 678 documents

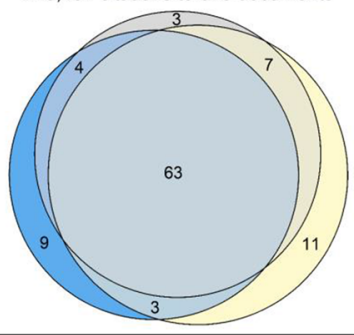

$\mathbf{F}$

Chemical \& Material Sciences

212,145 citations to 170 documents

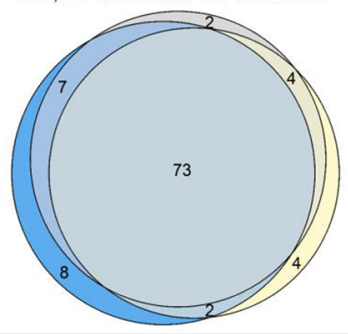

Fig. 6 Overlap between citations found by Scopus, Microsoft Academic, and Dimensions, by broad subject area. Figures within Venn diagrams expressed as percentages 


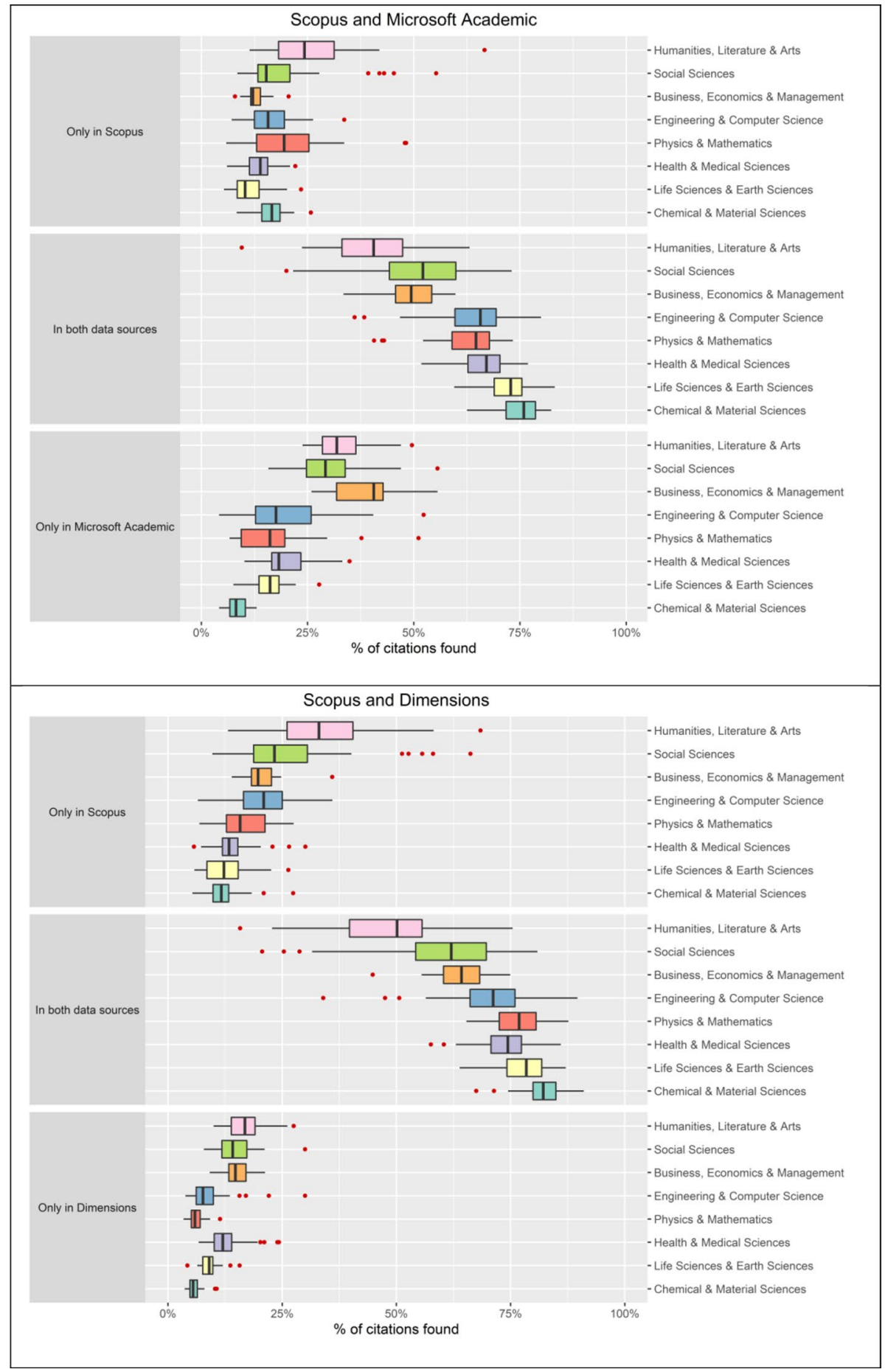

Fig. 7 Distribution of citations within each sector of the Venn diagrams that compare Scopus to Microsoft Academic and Dimensions. Calculated at the level of subject categories, and aggregated by subject areas 
the overlap in almost all cases exceeds 50\%. For Microsoft Academic/Scopus (Fig. 7, top), there are 66 (out of 252) subject categories where the overlap exceeds 70\%, and for Scopus/Dimensions, 148 categories exceed this overlap. Extreme cases of low overlap between sources are almost always in the Humanities and Social Sciences. For Microsoft Academic/ Scopus, the lowest overlaps (below 30\%) are in French Studies ${ }^{17}$ (9\%, although in this case the results are based only on citations to one seed document, because the rest were not covered by Microsoft Academic and Scopus), International Law ${ }^{18}$ (20\%), European Law ${ }^{19}$ (21\%), American Literature \& Studies ${ }^{20}$ (24\%), Law $^{21}$ (26\%), and Film 22 (27\%). In 182 categories (out of 252) Microsoft Academic found more citations than Scopus. There are also some outlier cases of low overlap in STEM categories, such as over $50 \%$ of citations in Computer Graphics ${ }^{23}$ and Discrete Mathematics ${ }^{24}$ only being available in Microsoft Academic (compared to Scopus), or $48 \%$ of citations in High Energy \& Nuclear Physics ${ }^{25}$ and Quantum Mechanics ${ }^{26}$ only being found by Scopus (compared to Microsoft Academic). For Scopus/Dimensions (Fig. 7, bottom), many of the same categories have the lowest overlap: French Studies,${ }^{27}$ International Law, ${ }^{28}$ American Literature \& Studies, ${ }^{29}$ European Law, ${ }^{30}$ and History. ${ }^{31}$ These low coverage figures are caused by Microsoft Academic and Dimensions having a lower coverage of citations in these categories than Scopus. In 36 categories (out of 252) Dimensions found more citations than Scopus.

\section{Web of Science and the new sources: Microsoft Academic and Dimensions}

Comparing Microsoft Academic, Dimensions and WoS (Fig. 8), there are many unique citations in Microsoft Academic and WoS. Out of these three, Dimensions found the fewest unique citations (2-6\% depending on the area). Again, the divergence is higher in the Humanities and Social Sciences, where Microsoft Academic has the highest percentages of unique citations. Microsoft Academic also has lower coverage in Physics \& Mathematics and (to a lower degree) in Chemical \& Material Sciences.

The results by subject category confirm that some categories deviate from the trend in a broad area (Fig. 9). Considering Microsoft Academic/WoS (Fig. 9, top), Microsoft Academic's coverage is large compared to WoS for Computing Systems ${ }^{32}$ (73\% of all citations),

17 https://osf.io/gmrju/.

18 https://osf.io/bzha2/.

19 https://osf.io/f36sn/.

20 https://osf.io/7qzmk/.

21 https://osf.io/4gtdc/.

22 https://osf.io/ctzb7/.

23 https://osf.io/rz4cj/.

24 https://osf.io/v6bgy/.

25 https://osf.io/vafzp/.

26 https://osf.io/87cdh/.

27 https://osf.io/bqpz4/.

28 https://osf.io/p26ua/.

29 https://osf.io/fngph/.

30 https://osf.io/pdnxt/.

31 https://osf.io/xjhfw/.

32 https://osf.io/ugvh3/. 
Web of Science $\bigcirc$ Microsoft Academic Dimensions

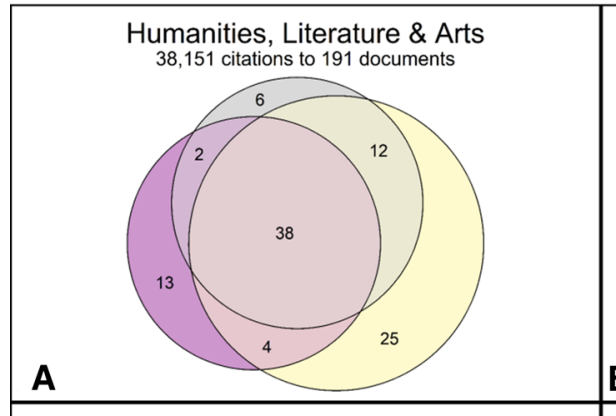

B

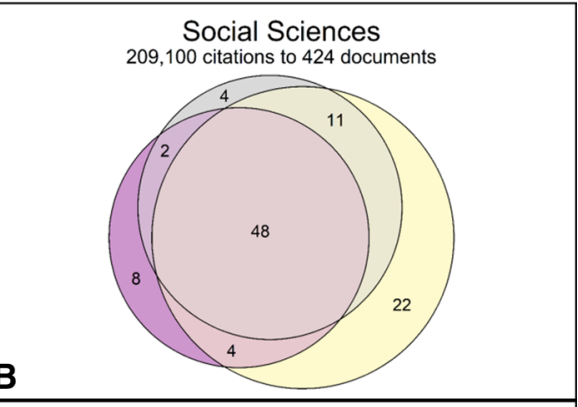
Business, Economics \& Management
123,203 citations to 144 documents

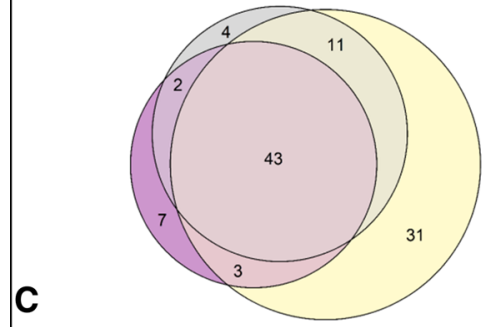

Physics \& Mathematics

228,769 citations to 221 documents

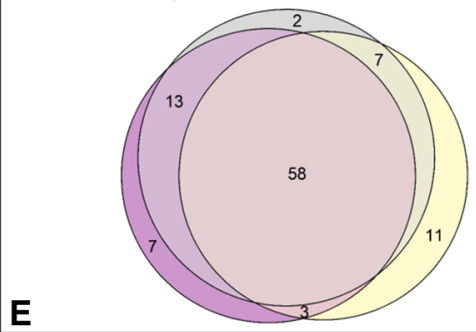

G

Life Sciences \& Earth Sciences 432,235 citations to 377 documents

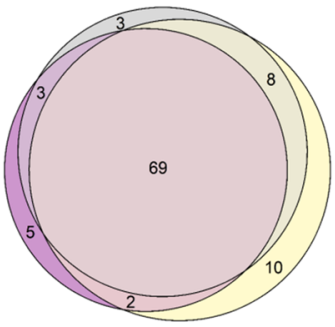

D

$\underset{445,912 \text { citations to } 502 \text { documents }}{\text { Engineering }}$

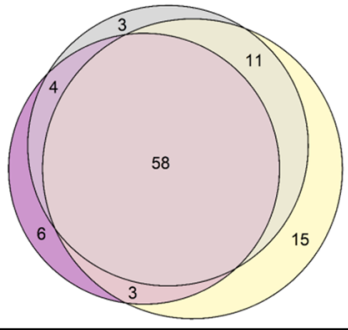

Health \& Medical Sciences 684,761 citations to 662 documents

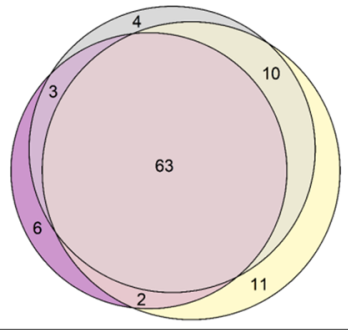

$\mathbf{F}$
Chemical \& Material Sciences 209,267 citations to 170 documents

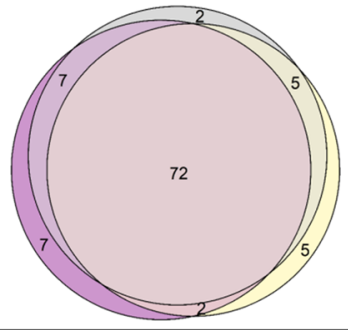

Fig. 8 Overlaps between citations found by Web of Science, Microsoft Academic, and Dimensions, by broad subject areas. Figures within Venn diagrams expressed as percentages 


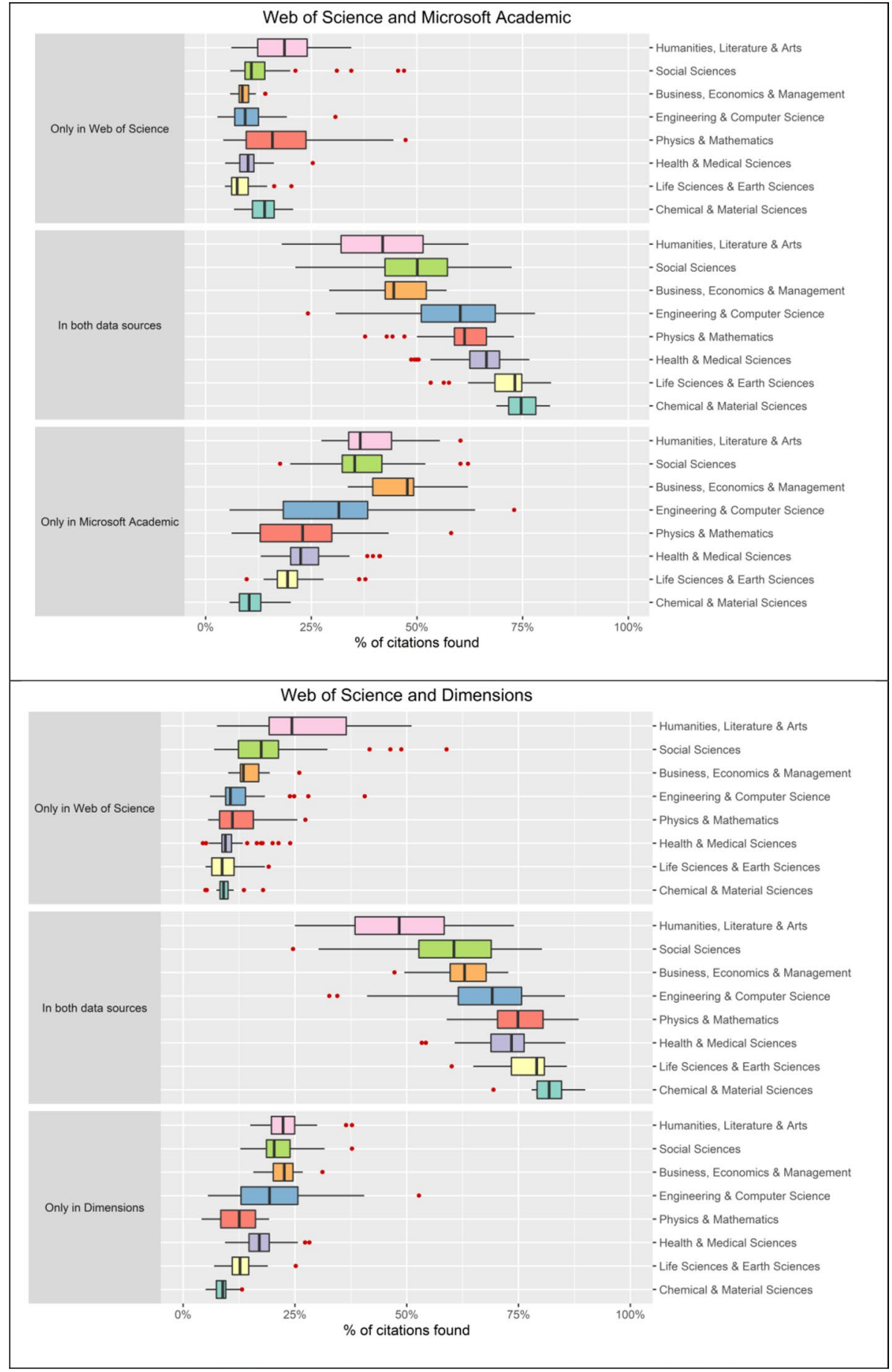

Fig. 9 Distribution of citations within each sector of the Venn diagrams that compare Web of Science to Microsoft Academic and Dimensions. Calculated at the level of subject categories, and aggregated by subject areas 
Software Systems ${ }^{33}$ (63\%), Educational Administration ${ }^{34}$ (62\%), Chinese Studies \& History ${ }^{35}$ (60\%), and Discrete Mathematics ${ }^{36}(58 \%)$. The gaps in coverage of Microsoft Academic in International Law, ${ }^{37}$ and $\mathrm{Law}^{38}$ occur again here, as $47 \%$ and $46 \%$ of the citations in these categories (respectively) are only found by WoS. Something similar occurs in the categories included in Physics \& Mathematics: the distribution of citations only found by WoS in this area has an unusually wide interquartile range when compared with the other areas, which is a sign that Microsoft Academic's gaps in coverage in this area affect more than one category. The most extreme cases are again Quantum Mechanics ${ }^{39}$ and High Energy \& Nuclear Phys$i c s,{ }^{40}$ with $47 \%$ and $44 \%$ of citations only found by WoS (respectively). In 223 categories (out of 252) Microsoft Academic found more citations than WoS. For the distributions of overlap and unique citations between Dimensions/WoS (Fig. 9, bottom), there are some similarities with the previous comparison: $51 \%$ of the citations in Computing Systems ${ }^{41}$ are only found by Dimensions, and in Humanities and Social Sciences over a third of the citations in Chinese Studies \& History, ${ }^{42}$ and Foreign Language Learning ${ }^{43}$ are only found by Dimensions, which reveals coverage gaps in these categories in WoS. In other Humanities categories, such as American Literature \& Studies ${ }^{44}(51 \%)$, History ${ }^{45}(46 \%)$, or Literature \& Writing ${ }^{46}(46 \%)$ WoS found more unique citations than Dimensions. Dimensions also has gaps in coverage in Computer Graphics, ${ }^{47}$ International Law, ${ }^{48}$ Law, ${ }^{49}$ and Middle Eastern \& Islamic Studies, ${ }^{50}$ compared to WoS. In 185 categories (out of 252) Dimensions found more citations than WoS.

\section{Microsoft Academic and Dimensions}

At the level of subject categories, the vast majority of citations in Microsoft Academic/ Dimensions are found either by both databases, or only by Microsoft Academic. In 209 out of 252 categories, the percentage of unique citations in Dimensions is below 10\% (Fig. 10). The exceptions are in Physics \& Mathematics, where $45 \%$ of the citations in Quantum Mechanics, ${ }^{51} 39 \%$ of the citations in High Energy \& Nuclear Physics, ${ }^{52}$ and $26 \%$ of the

\footnotetext{
33 https://osf.io/6vrnp/.

34 https://osf.io/x9g3e/.

35 https://osf.io/54xky/.

36 https://osf.io/fa8sr/.

37 https://osf.io/9584j/.

38 https://osf.io/h7jt2/.

39 https://osf.io/ghws2/.

40 https://osf.io/gpyse/.

41 https://osf.io/rsj4m/.

42 https://osf.io/bvr3p/.

43 https://osf.io/vmdbx/.

44 https://osf.io/zd53e/.

45 https://osf.io/q529p/.

46 https://osf.io/qcdsh/.

47 https://osf.io/sfd2g/.

48 https://osf.io/a9mtx/.

49 https://osf.io/n2e98/.

50 https://osf.io/za5ks/.

51 https://osf.io/3npwu/.

52 https://osf.io/7qb8v/.
} 


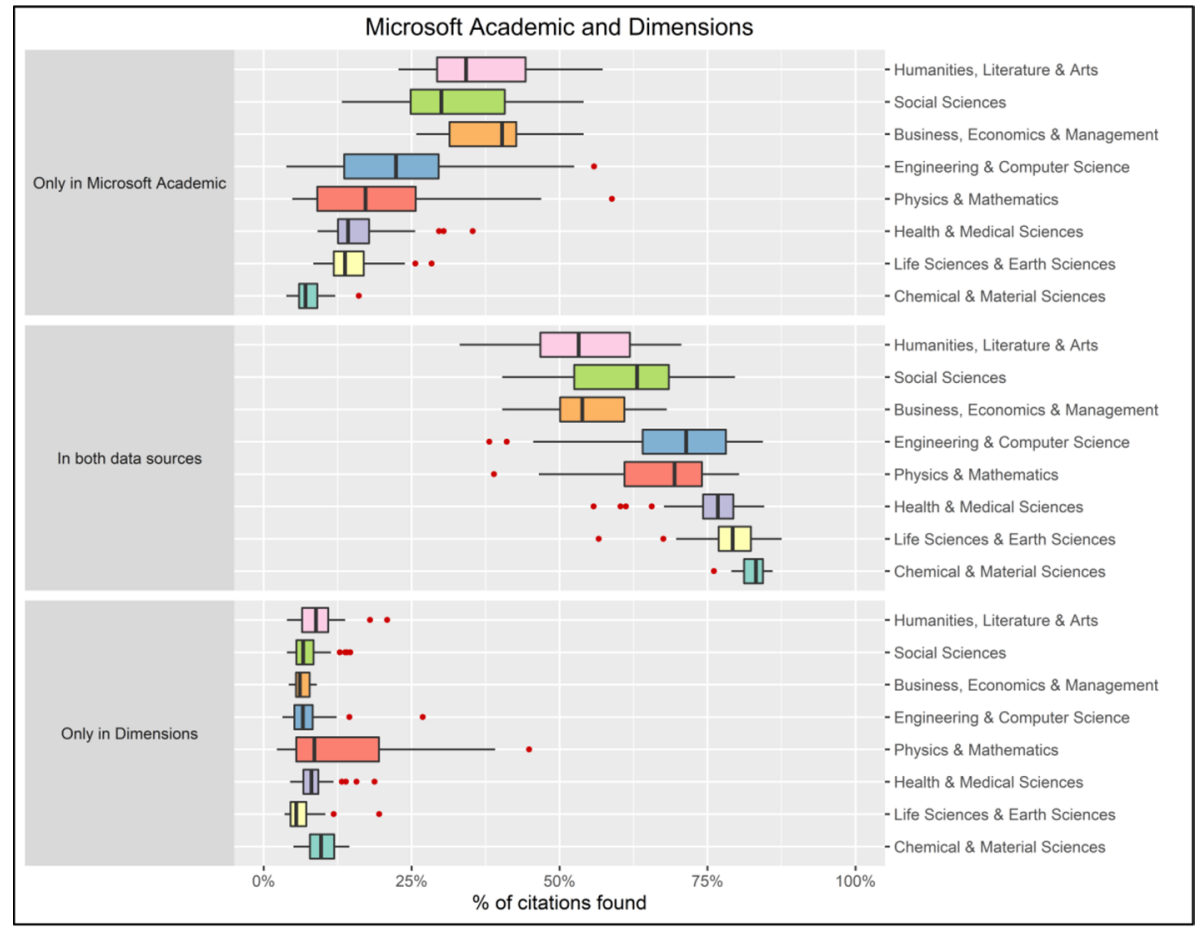

Fig. 10 Distribution of citations within each sector of the Venn diagrams that compare Microsoft Academic and Dimensions. Calculated at the level of subject categories, and aggregated by subject areas

citations in Plasma \& Fusion ${ }^{53}$ (also included in Engineering \& Computer Science) are only found by Dimensions. This again reveals the gap in coverage of Microsoft Academic in these categories. In 226 categories (out of 252) Microsoft Academic found more citations than Dimensions.

\section{Discussion}

\section{Limitations}

Because this study uses an updated and extended version of the sample used in MartínMartín et al. (2018), many of the limitations declared in that study are also applicable here, as summarised below.

- The seed sample of documents used all highly-cited documents published in English in 2006 according to Google Scholar's Classic Papers product. To generalize the results presented here, it must be assumed that the population of documents that cite these

53 https://osf.io/n5j8v/. 
highly-cited documents is comparable to the general population of citing documents within each subject category. This might not be true in some cases, because different topics within the same category might have different citation patterns (certain highlycited topics within a category might be overrepresented). Also, these results probably do not represent the reality of coverage of academic literature published in languages other than English and literature about regionally-relevant topics, where Google Scholar, Microsoft Academic, Dimensions and COCI may have an advantage.

- Google Scholar might have an unfair advantage in this analysis, as the initial seed sample was selected from a list of the highest-cited documents in this source (the accuracy of citation detection of Google Scholar in this specific sample could be higher than the average accuracy of citation detection across all documents in Google Scholar, which is unknown). However, the correlations between the citation counts of citing documents available in Martín-Martín et al. (2018) suggest that this advantage is not substantial: when analysing documents from the entire distribution of citation counts (not only highly-cited documents), Google Scholar still consistently reports higher citation counts than WoS and Scopus, while providing essentially the same citation rankings at the document level in most subject categories as the other two sources.

- The algorithm that matches citations across data sources is intentionally conservative: it is set to minimize false positives, potentially at the expense of false negatives. Therefore, the percentages of overlap shown in this study are lower bounds.

- Unlike Martín-Martín et al. (2018), where citations from documents included in the ESCI Backfile for documents published between 2005 and 2014 were not included in the analysis, in this study all available citation data available in the citation indexes that are part of WoS Core Collection is analysed.

- Data extraction for this analysis was carried out in May/June 2019. However, the rapid development of these platforms may render the results obsolete in the future. Updated analyses similar to this one might be necessary to ascertain the current coverage of the data sources, especially if regular reports on coverage development are not issued by the sources themselves.

Other aspects related to coverage, such as the distribution of document types, language, date of publication, or indexing speed are not analysed here and could be investigated in future studies, as they are also necessary for users who need to decide which data source(s) are most suitable for their needs.

\section{Comparison with previous studies}

The results generally agree with previous studies comparing the coverage of Microsoft Academic and Dimensions. Similarly to Harzing \& Alakangas (2017a, b) and Thelwall (2017), Microsoft Academic detected more citations than WoS and Scopus. This citation detection advantage seems to be higher in the Humanities, Social Sciences, and Business $\&$ Economics than in the other areas, where in some cases Microsoft Academic had lower coverage (Physics, Chemistry). The results here cannot be directly compared to Hug and Brändle (2017), who reported that Scopus had slightly greater coverage of journal articles than Microsoft Academic, because this study does not analyse specific document types. However, assuming that most citations come from journal articles, Microsoft Academic seems to have now surpassed Scopus in raw size, at least in the three areas mentioned above. 
For Dimensions, the results also agree with those reported by Harzing (2019), who found that it had a similar or better coverage than WoS and Scopus in Business \& Economics. Here the results show that the three data sources offer a similar coverage (Scopus is slightly larger, followed by Dimensions), but each can detect a non-negligible proportion of citations that the others can't.

From Visser et al. (2020) the percentage of documents covered by Scopus that are also covered by Dimensions is $78 \%$, but in this study the percentage of citations found by Scopus that are also found by Dimensions is higher (84\%). The causes of the difference between these figures is unclear, but some possibilities are (a) this study uses a sample of citations while Visser et al. use the entire collection of source documents, (b) the possibility that Dimensions has a lower coverage of older documents (our study analyses citations from 2006 to 2018, while Visser et al. analysed coverage between 1996 and 2017), or (c) that there was an increase in coverage between the time Visser et al. obtained their data (December 2018), and the time the data for this study was extracted (May-June 2019). The overlap Visser et al. found between Scopus and WoS is significantly lower than found here: according to their results (overlap of 29.1 million documents, and 44.9 million documents in total in Scopus), WoS covered $65 \%$ of the documents available in Scopus. In the current study, however, WoS found $83 \%$ of the citations found by Scopus. The cause of this significant difference is also unknown, but it might be in part caused by the fact that Visser et al. analysed only documents in the SCI, SSCI, and A\&HCI and the Conference Proceedings Citation Index (CPCI), while this study also considers other citation indexes within WoS Core Collection, such as ESCI and BKCI. Lastly, the percentage of Scopus documents also covered by Microsoft Academic reported by Visser et al. (81\%) is very similar to the percentage of Scopus citations also found by Microsoft Academic reported here (82\%). However, the full overlap between the two sources is much higher here $(66 \%)$ than in Visser et al. (18\%), because in the latter study a much higher amount of unique content was detected in Microsoft Academic. One possible reason for this might be that our study only considers documents with recorded relationships to other documents (through citations), while some of the documents in Microsoft Academic analysed in Visser et al. might not have these connections, which would make them undetectable to our methodology.

Although most of the results of the overlap analysis reported here closely match those of the previous study with the same seed set (Martín-Martín et al. 2018), several discrepancies were found. In two subject categories (Psychology, and Astronomy \& Astrophysics), the updated analysis showed that Google Scholar had a lower coverage than the other data sources, while in the old dataset, this was not the case. In the case of Astronomy \& Astrophysics, this apparent fluctuation in coverage is consistent with an editorial published in August of 2019 in the journal Astronomy \& Astrophysics, which denounced a sharp decrease in the h5-index of this journal in the 2019 edition of Google Scholar Metrics (Forveille 2019) caused by a technical error in Google Scholar. Therefore, this seems to be a new case of a major coverage outage in Google Scholar, similar to one previously reported by Delgado López-Cózar and MartínMartín (2018) which affected many journals published in Spain, and which was resolved when Google Scholar rebuilt its index a few months later. This issue will be analysed in detail in a future study as an example of how coverage in Google Scholar can suffer large (downward) fluctuations over time, as this can negatively affect literature search. 


\section{Conclusions}

\section{Comprehensiveness of data sources across subject categories}

The results show that Google Scholar is still the most comprehensive data source among the six studied here. This holds true for the overall results and the results across all subject areas, with some exceptions such as Astronomy \& Astrophysics. Google Scholar found nearly all the citations found by Microsoft Academic, Dimensions, and COCI (89\%, 93\%, and $94 \%$, respectively). The largest divergences occur in the Humanities and Social Sciences (lowest value is $84 \%$, which corresponds to the percentage of Scopus citations in the Humanities found by Google Scholar). Additionally, there is a significant amount of extra coverage in Google Scholar that is not found in any of the other data sources ( $26 \%$ of all citations across all data sources). Google Scholar could therefore make an important contribution to the scientific community by opening its bibliographic and citation data, which would also facilitate the identification of errors such as coverage fluctuations.

Whilst the results confirm that Microsoft Academic and Dimensions provide at least as many citations as Scopus and WoS in many subject categories, some gaps still exist:

- Microsoft Academic seems to index the Humanities, Social Sciences, and Business, Economics \& Management particularly well, although not for all categories.

- Dimensions is closely behind Scopus in all areas in terms of citations found, but surpasses WoS in all areas, except in two (Physics \& Mathematics, and Chemical \& Material Sciences) where they are tied, although there are also differences at the level of subject categories (Dimensions also has coverage gaps in some Humanities categories).

\section{Implications for academic literature search}

Although Google Scholar and Microsoft Academic are the two most comprehensive bibliographic data sources analysed in this study, their search functionalities have a number of limitations, such as limited support of Boolean and other types of search operators, limited filtering capabilities (Google Scholar), and non-transparent algorithms to process queries and rank the documents in the results page (Microsoft Academic uses artificial intelligence, and Google Scholar uses publicly unknown heuristics to rank documents by relevance) (Beel and Gipp 2009a, b, c; Martin-Martin et al. 2017; Orduña-Malea et al. 2016; Rovira et al. 2019; Wang et al. 2020). These characteristics, which prevent users from being able to generate complex search equations that are guaranteed to stay reproducible over time, have led some authors to consider Google Scholar and Microsoft Academic inadequate for query-based search (Gusenbauer and Haddaway 2020). Dimensions, which does not allow complex Boolean searches in its web interface either, was not analysed in that study.

On the other hand, Scopus and WoS have a lower coverage, especially in some areas such as the Humanities and Social Sciences, do not cover non-peer-reviewed scientific documents (Martín-Martín et al. 2018), are slower at indexing (Moed et al. 2016), and are not free. These characteristics reduce their usefulness in situations where fast and unrestricted access to the latest studies is important, such as the COVID-19 pandemic in which preprints play a critical role (Fraser et al. 2020). Nevertheless, these sources offer advanced search and filtering functionalities, and were considered suitable tools for evidence synthesis in the form of systematic reviews (Gusenbauer and Haddaway 2020). 
Thus, there seems to be a mismatch between the bibliographic data sources that are currently the most comprehensive, and those that offer users the most control over their searches. Since systematic reviews require both comprehensiveness of coverage and control over the search process, it is possible that in some cases no single currently available data source is adequate for the task, and instead at least two sources should be used. One way to do this would be to expand the concept of systematic search beyond the traditional search query to include other non-query-based search processes that can also be carried out in a systematic and reproducible manner. One possibility would be the expansion of a document collection obtained in a query-based search through the analysis of its citation network. This expansion can be carried out in a more comprehensive data source, different from the one where the initial search was carried out. As a longer term solution, academic search tools should strive to offer more a transparent and reproducible search process and embrace community standards for interoperability and reuse of document metadata (Haddaway and Gusenbauer 2020).

Lastly, searches suitable for systematic reviews are only one of the many types of search that are carried out in these data sources. Indeed, the more recent academic search platforms (Microsoft Academic, Semantic Scholar, Dimensions) have not implemented traditional advanced query-based capabilities (Dimensions supports them in its API), and seem to be instead focusing on the browsing experience (advanced filtering), and in offering analytics dashboards. Lens.org seems to be an exception, as it also offers advanced structured query-based search (Tay 2019). Future studies could focus on the suitability of these and other bibliographic data sources to solve specific types of information needs, as it is important that researchers are aware of the strengths and limitations of each data source for specific use cases and in specific knowledge domains.

\section{Implications for bibliometric analyses}

As new sources of bibliographic data (including citation data) become openly available and validated for specific types of bibliometric analyses, the need to rely on expensive proprietary data sources may diminish. Regarding the findings in this study, the final decision about which source to use may depend on properties of the sources other than coverage, such as metadata quality and bulk access options. If these factors are not of overriding importance, however, then Google Scholar is the best choice in almost all subject areas for those needing the most comprehensive citation counts but not needing complete lists of citing sources. If complete lists are needed, then Microsoft Academic is the best alternative and is also free. The amount of citation data in the public domain (through COCI) is still low and not useful on its own, presumably because its role is to feed other sources, not to be more comprehensive than them.

In use cases where exhaustiveness of coverage is required, but coverage divergence is considered to be large (many unique citations in each data source), the combination of several sources is recommended.

To conclude, the evidence presented in this study is intended to serve as a tool for researchers and other users of bibliographic databases, one that will hopefully help them make more informed decisions when they need to select one or more of these data sources to solve a specific information need. 
Acknowledgements We thank Medialab UGR (Universidad de Granada) for providing funding to cover the cost of hosting the interactive web application ${ }^{54}$ created to explore the data used in this study. We thank Digital Science for providing free access to the Dimensions API. We thank Jing Xuan Xie for translating the abstract to Chinese. We thank Asura Enkhbayar for suggesting the use of an upset plot in Fig. 2. Lastly, we thank two anonymous reviewers for their thoughtful comments, which have helped improved the manuscript substantially.

\section{Appendix 1: Complete list of Venn diagrams computed for this study}

No subject aggregation

Two-set Venn diagrams (all subject categories)

https://osf.io/bwpaq/

Three-set Venn diagrams (all subject categories) https://osf.io/jkrge/

Aggregated by 8 subject areas

Google Scholar-Microsoft Academic-Scopus

https://osf.io/h7m8s/

Google Scholar-Microsoft Academic-Dimensions

https://osf.io/7v4kr/

Google Scholar-Microsoft Academic-Web of Science

https://osf.io/fn3yh/

Google Scholar-Microsoft Academic-COCI

https://osf.io/s3bmp/

Google Scholar-Scopus-Dimensions

https://osf.io/q8ecx/

Google Scholar-Scopus-Web of Science

https://osf.io/qkc2a/

Google Scholar-Scopus-COCI

https://osf.io/mrvdb/

Google Scholar-Dimensions-Web of Science

https://osf.io/nwm83/

Google Scholar-Dimensions-COCI

https://osf.io/dzs5x/

Google Scholar-Web of Science-COCI

https://osf.io/64chg/

Microsoft Academic-Scopus-Dimensions

https://osf.io/hgzn6/

Microsoft Academic-Scopus-Web of Science

https://osf.io/f7xpa/

Microsoft Academic-Scopus-COCI

https://osf.io/c6tpz/

Microsoft Academic-Dimensions-Web of Science

https://osf.io/f5zxs/

Microsoft Academic-Dimensions-COCI

https://osf.io/ry87a/

Microsoft Academic-Web of Science-COCI

https://osf.io/vxyj4/

Scopus-Dimensions-Web of Science

https://osf.io/xqg3y/

Scopus-Dimensions-COCI

https://osf.io/jmvb6/

Aggregated by 252 subject categories (zipped)

Google Scholar-Microsoft Academic

https://osf.io/v4ek3/

Google Scholar-Scopus

https://osf.io/umsyw/

Google Scholar-Dimensions

https://osf.io/jqmuy/

Google Scholar-Web of Science

https://osf.io/4b8uq/

Google Scholar-COCI

https://osf.io/gytuh/

Microsoft Academic-Scopus

https://osf.io/jw2bt/

Microsoft Academic-Dimensions

https://osf.io/a2mp7/

Microsoft Academic-Web of Science

https://osf.io/2hkxq/

Microsoft Academic-COCI

https://osf.io/ch4gb/

54 https://albertomartin.shinyapps.io/citation_overlap_2019/. 


\begin{tabular}{ll}
\hline Aggregated by 252 subject categories (zipped) & \\
\hline Scopus-Dimensions & https://osf.io/q4swk/ \\
Scopus-Web of Science & https://osf.io/qcpbh/ \\
Scopus-COCI & https://osf.io/2xvbh/ \\
Dimensions-Web of Science & https://osf.io/pdycb/ \\
Dimensions-COCI & https://osf.io/j7qte/ \\
Web of Science-COCI & https://osf.io/mnwe7/
\end{tabular}

\section{Appendix 2: Complete list of boxplots computed for this study}

\begin{tabular}{ll}
\hline Subject category-level overlap data aggregated by 8 subject areas & \\
\hline Google Scholar-Microsoft Academic & https://osf.io/b94xp/ \\
Google Scholar-Scopus & https://osf.io/rvbw9/ \\
Google Scholar-Dimensions & https://osf.io/ubtrm/ \\
Google Scholar-Web of Science & https://osf.io/7wb49/ \\
Google Scholar-COCI & https://osf.io/7ekdr/ \\
Microsoft Academic-Scopus & https://osf.io/jx7by/ \\
Microsoft Academic-Dimensions & https://osf.io/x4257/ \\
Microsoft Academic-Web of Science & https://osf.io/rdw7g/ \\
Microsoft Academic-COCI & https://osf.io/f8a9e/ \\
Scopus-Dimensions & https://osf.io/3a97k/ \\
Scopus-Web of Science & https://osf.io/w4zv3/ \\
Scopus-COCI & https://osf.io/jtnyu/ \\
Dimensions-Web of Science & https://osf.io/gsjwm/ \\
Dimensions-COCI & https://osf.io/sr4wu/ \\
Web of Science - COCI & https://osf.io/6dkw4/ \\
\hline
\end{tabular}

\section{References}

Baas, J., Schotten, M., Plume, A., Côté, G., \& Karimi, R. (2020). Scopus as a curated, high-quality bibliometric data source for academic research in quantitative science studies. Quantitative Science Studies, 1(1), 377-386. https://doi.org/10.1162/qss_a_00019.

Beel, J., \& Gipp, B. (2009a). Google Scholar's ranking algorithm: The impact of articles' age (an empirical study). Sixth International Conference on Information Technology: New Generations, 2009, 160-164. https://doi.org/10.1109/ITNG.2009.317.

Beel, J., \& Gipp, B. (2009b). Google Scholar's ranking algorithm: The impact of citation counts (An empirical study). Third International Conference on Research Challenges in Information Science, 2009, 439-446. https://doi.org/10.1109/RCIS.2009.5089308.

Beel, J., \& Gipp, B. (2009c). Google Scholar's ranking algorithm: An introductory overview. In Proceedings of the 12th International Conference on Scientometrics and Informetrics (ISSI'09) (pp. 230-241). http://www.issi-society.org/proceedings/issi_2009/ISSI2009-proc-vol1_Aug2009_batch2-paper-1.pdf

Birkle, C., Pendlebury, D. A., Schnell, J., \& Adams, J. (2020). Web of Science as a data source for research on scientific and scholarly activity. Quantitative Science Studies, 1(1), 363-376. https://doi. org/10.1162/qss_a_00018. 
Chapman, K., \& Ellinger, A. E. (2019). An evaluation of Web of Science, Scopus and Google Scholar citations in operations management. The International Journal of Logistics Management, 30(4), 10391053. https://doi.org/10.1108/IJLM-04-2019-0110.

Damerau, F. J. (1964). A technique for computer detection and correction of spelling errors. Communications of the ACM, 7(3), 171-176. https://doi.org/10.1145/363958.363994.

Delgado López-Cózar, E., \& Martín-Martín, A. (2018). Apagón digital de la producción científica española en Google Scholar. Anuario ThinkEPI, 12, 265-276. https://doi.org/10.3145/thinkepi.2018.40.

Delgado López-Cózar, E., Orduna-Malea, E., \& Martín-Martín, A. (2019). Google Scholar as a data source for research assessment. In W. Glaenzel, H. Moed, U. Schmoch, \& M. Thelwall (Eds.), Springer handbook of science and technology indicators. Berlin: Springer.

Dowle, M., Srinivasan, A., Gorecki, J., Chirico, M., Stetsenko, P., Short, T., Lianoglou, S., Antonyan, E., Bonsch, M., \& Parsonage, H. (2018). data.table: Extension of 'data.frame' (1.11.4).

Else, H. (2018, April 11). How I scraped data from Google Scholar. Nature. https://doi.org/10.1038/d4158 6-018-04190-5

Forveille, T. (2019). A\&A ranking by Google. Astronomy \& Astrophysics, 628, E1. https://doi. org/10.1051/0004-6361/201936429.

Fraser, N., Brierley, L., Dey, G., Polka, J. K., Pálfy, M., \& Coates, J. A. (2020). Preprinting a pandemic: The role of preprints in the COVID-19 pandemic. BioRxiv, 2020.05.22.111294. https://doi. org/10.1101/2020.05.22.111294

Gusenbauer, M. (2018). Google Scholar to overshadow them all? Comparing the sizes of 12 academic search engines and bibliographic databases. Scientometrics. https://doi.org/10.1007/s11192-018-2958-5.

Gusenbauer, M., \& Haddaway, N. R. (2020). Which academic search systems are suitable for systematic reviews or meta-analyses? Evaluating retrieval qualities of Google Scholar, PubMed, and 26 other resources. Research Synthesis Methods, 11(2), 181-217. https://doi.org/10.1002/jrsm.1378.

Haddaway, N., \& Gusenbauer, M. (2020, February 3). A broken system: Why literature searching needs a FAIR revolution. Impact of Social Sciences. https://blogs.lse.ac.uk/impactofsocialsciences/2020/02/03/ a-broken-system-why-literature-searching-needs-a-fair-revolution/.

Halevi, G., Moed, H., \& Bar-Ilan, J. (2017). Suitability of Google Scholar as a source of scientific information and as a source of data for scientific evaluation-Review of the Literature. Journal of Informetrics, 11(3), 823-834. https://doi.org/10.1016/J.JOI.2017.06.005.

Harzing, A. W. (2016). Microsoft Academic (Search): A Phoenix arisen from the ashes? In Scientometrics (Vol. 108, No. 3, pp. 1637-1647). Springer, Netherlands. https://doi.org/10.1007/s11192-016-2026-y

Harzing, A.-W. (2016). Sacrifice a little accuracy for a lot more comprehensive coverage. Harzing.Com. https://harzing.com/blog/2016/08/sacrifice-a-little-accuracy-for-a-lot-more-comprehensive-coverage

Harzing, A. W. (2019). Two new kids on the block: How do Crossref and Dimensions compare with Google Scholar, Microsoft Academic, Scopus and the Web of Science? In Scientometrics (Vol. 120, Issue 1, pp. 341-349). Springer, Netherlands. https://doi.org/10.1007/s11192-019-03114-y

Harzing, A.-W., \& Alakangas, S. (2016). Google Scholar, Scopus and the Web of Science: A longitudinal and cross-disciplinary comparison. Scientometrics, 106(2), 787-804. https://doi.org/10.1007/s1119 2-015-1798-9.

Harzing, A. W., \& Alakangas, S. (2017a). Microsoft Academic: Is the phoenix getting wings? In Scientometrics (Vol. 110, Issue 1, pp. 371-383). Springer, Netherlands. https://doi.org/10.1007/s1119 2-016-2185-X

Harzing, A. W., \& Alakangas, S. (2017b). Microsoft Academic is one year old: The Phoenix is ready to leave the nest. In Scientometrics (Vol. 112, Issue 3, pp. 1887-1894). Springer, Netherlands. https://doi. org/10.1007/s11192-017-2454-3

Haunschild, R., Hug, S. E., Brändle, M. P., \& Bornmann, L. (2018). The number of linked references of publications in Microsoft Academic in comparison with the Web of Science. In Scientometrics (Vol. 114, Issue 1, pp. 367-370). Springer, Netherlands. https://doi.org/10.1007/s11192-017-2567-8

Heibi, I., Peroni, S., \& Shotton, D. (2019). Software review: COCI, the OpenCitations Index of Crossref open DOI-to-DOI citations. Scientometrics. https://doi.org/10.1007/s11192-019-03217-6.

Hendricks, G., Tkaczyk, D., Lin, J., \& Feeney, P. (2020). Crossref: The sustainable source of communityowned scholarly metadata. Quantitative Science Studies, 1(1), 414-427. https://doi.org/10.1162/ qss_a_00022.

Herzog, C., Hook, D., \& Konkiel, S. (2020). Dimensions: Bringing down barriers between scientometricians and data. Quantitative Science Studies, 1(1), 387-395. https://doi.org/10.1162/qss_a_00020.

Hook, D. W., Porter, S. J., \& Herzog, C. (2018). Dimensions: Building Context for Search and Evaluation. Frontiers in Research Metrics and Analytics, 3, 23. https://doi.org/10.3389/frma.2018.00023. 
Huang, C.-K., Neylon, C., Brookes-Kenworthy, C., Hosking, R., Montgomery, L., Wilson, K., et al. (2020). Comparison of bibliographic data sources: Implications for the robustness of university rankings. Quantitative Science Studies. https://doi.org/10.1162/qss_a_00031.

Hug, S. E., \& Brändle, M. P. (2017). The coverage of Microsoft Academic: Analyzing the publication output of a university. Scientometrics, 113(3), 1551-1571. https://doi.org/10.1007/s11192-017-2535-3.

Kousha, K., \& Thelwall, M. (2018). Can Microsoft Academic help to assess the citation impact of academic books? Journal of Informetrics, 12(3), 972-984. https://doi.org/10.1016/j.joi.2018.08.003.

Kousha, K., Thelwall, M., \& Abdoli, M. (2018). Can Microsoft Academic assess the early citation impact of in-press articles? A multi-discipline exploratory analysis. Journal of Informetrics, 12(1), 287-298. https://doi.org/10.1016/j.joi.2018.01.009.

Krassowski, M. (2020). ComplexUpset. https://github.com/krassowski/complex-upset

Larsson, J., Godfrey, A. J. R., Kelley, T., Eberly, D. H., Gustafsson, P., \& Huber, E. (2018). eulerr: AreaProportional Euler and Venn Diagrams with Circles or Ellipses (4.1.0).

Levenshtein, V. I. (1966). Binary codes capable of correcting deletions, insertions, and reversals. Soviet Physics Doklady, 10(8), 707-710.

Martín-Martín, A. (2018). Code to extract bibliographic data from Google Scholar (v1.0). Zenodo. https:// doi.org/10.5281/zenodo.1481076

Martín-Martín, A., \& Delgado López-Cózar, E. (2016). Reading Web of Science data into R (0.6).

Martin-Martin, A., Orduna-Malea, E., Harzing, A.-W., \& Delgado López-Cózar, E. (2017). Can we use Google Scholar to identify highly-cited documents? Journal of Informetrics, 11(1), 152-163. https:// doi.org/10.1016/j.joi.2016.11.008.

Martín-Martín, A., Orduna-Malea, E., Thelwall, M., \& Delgado López-Cózar, E. (2018). Google Scholar, Web of Science, and Scopus: A systematic comparison of citations in 252 subject categories. Journal of Informetrics, 12(4), 1160-1177. https://doi.org/10.1016/J.JOI.2018.09.002.

Moed, H. F., Bar-Ilan, J., \& Halevi, G. (2016). A new methodology for comparing Google Scholar and Scopus. Journal of Informetrics, 10(2), 533-551. https://doi.org/10.1016/j.joi.2016.04.017.

Orduña-Malea, E., \& Delgado-López-Cózar, E. (2018). Dimensions: Re-discovering the ecosystem of scientific information. Profesional de La Informacion, 27(2), 420-431. https://doi.org/10.3145/epi.2018. mar.21.

Orduña-Malea, E., Martín-Martín, A., Ayllon, M., \& Delgado López-Cózar, E. (2014). The silent fading of an academic search engine: The case of Microsoft Academic Search. Online Information Review, 38(7), 936-953. https://doi.org/10.1108/OIR-07-2014-0169.

Orduña-Malea, E., Martín-Martín, A., Ayllón, J. M., \& Delgado López-Cózar, E. (2016). La revolución Google Scholar: Destapando la caja de Pandora académica. Universidad de Granada y Unión de Editoriales Universitarias Españolas.

Orduna-Malea, E., Martín-Martín, A., \& Delgado López-Cózar, E. (2017). Google Scholar as a source for scholarly evaluation: A bibliographic review of database errors. Revista Española de Documentación Científica, 40(4), e185. https://doi.org/10.3989/redc.2017.4.1500.

Orduna-Malea, E., Martín-Martín, A., \& Delgado López-Cózar, E. (2018). Classic papers: Using Google Scholar to detect the highly-cited documents. In 23rd International conference on science and technology indicators (pp. 1298-1307). https://doi.org/10.31235/osf.io/zkh7p

Ortega, J. L. (2014). Academic search engines: A quantitative outlook. Cambridge: Chandos Publishing.

Peroni, S., \& Shotton, D. (2020). OpenCitations, an infrastructure organization for open scholarship. Quantitative Science Studies, 1(1), 428-444. https://doi.org/10.1162/qss_a_00023.

R Core Team. (2014). R: A Language and Environment for Statistical Computing.

Rovira, C., Codina, L., Guerrero-Solé, F., \& Lopezosa, C. (2019). Ranking by relevance and citation counts, a comparative study: Google Scholar, Microsoft academic, WoS and scopus. Future Internet, 11(9), 202. https://doi.org/10.3390/fi11090202.

Shotton, D. (2013). Publishing: Open citations. Nature, 502(7471), 295-297. https://doi.org/10.1038/50229 5 a.

Shotton, D. (2018). Funders should mandate open citations. Nature, 553(7687), 129. https://doi.org/10.1038/ d41586-018-00104-7.

Tay, A. (2019, April 3). 6 reasons why you should try Lens.org. Medium. https://medium.com/@aarontay/6reasons-why-you-should-try-lens-org-c40abb09ec6f

Thelwall, M. (2017). Microsoft Academic: A multidisciplinary comparison of citation counts with Scopus and Mendeley for 29 journals. Journal of Informetrics, 11(4), 1201-1212. https://doi.org/10.1016/j. joi.2017.10.006.

Thelwall, M. (2018a). Does Microsoft Academic find early citations? Scientometrics, 114(1), 325-334. https://doi.org/10.1007/s11192-017-2558-9. 
Thelwall, M. (2018b). Microsoft Academic automatic document searches: Accuracy for journal articles and suitability for citation analysis. Journal of Informetrics, 12(1), 1-9. https://doi.org/10.1016/j. joi.2017.11.001.

Thelwall, M. (2018c). Dimensions: A competitor to Scopus and the Web of Science? Journal of Informetrics, 12(2), 430-435. https://doi.org/10.1016/j.joi.2018.03.006.

van der Loo, M., van der Laan, J., R Core Team, Logan, N., \& Muir, C. (2018). stringdist: Approximate String Matching and String Distance Functions (0.9.5.1).

van Eck, N. J., \& Waltman, L. (2019). Accuracy of citation data in Web of Science and Scopus.

van Eck, N. J., Waltman, L., Larivière, V., \& Sugimoto, C. (2018). Crossref as a new source of citation data: A comparison with Web of Science and Scopus. https://www.cwts.nl/blog?article=n-r2s234\&title $=$ crossref-as-a-new-source-of-citation-data-a-comparison-with-web-of-science-and-scopus

Van Noorden, R. (2014). November 7). Google Scholar pioneer on search engine's future. Nature.. https:// doi.org/10.1038/nature.2014.16269.

Visser, M., van Eck, N. J., \& Waltman, L. (2020). Large-scale comparison of bibliographic data sources: Scopus, Web of Science, Dimensions, Crossref, and Microsoft Academic. https://arxiv.org/ abs/2005.10732

Walker, A., \& Braglia, L. (2018). openxlsx: Read, Write and Edit XLSX Files (4.1.0).

Wang, K., Shen, Z., Huang, C., Wu, C.-H., Dong, Y., \& Kanakia, A. (2020). Microsoft academic graph: When experts are not enough. Quantitative Science Studies, 1(1), 396-413. https://doi.org/10.1162/ qss_a_00021.

Wickham, H. (2016). ggplot2: Elegant graphics for data analysis. New York: Springer.

Wilke, C. O. (2019). cowplot: Streamlined Plot Theme and Plot Annotations for 'ggplot2'.

Wu, J., Kim, K., \& Giles, C. L. (2019). CiteSeerX: 20 years of service to scholarly big data. Proceedings of the Conference on Artificial Intelligence for Data Discovery and Reuse. https://doi.org/10.1145/33591 15.3359119 . 